\title{
Influence of the hydrodynamic drag from an accretion torus on extreme mass-ratio inspirals
}

\author{
Enrico Barausse* \\ SISSA, International School for Advanced Studies and INFN, Via Beirut 2, 34014 Trieste, Italy \\ Luciano Rezzolla \\ Max-Planck-Institut für Gravitationsphysik, Albert-Einstein-Institut, 14476 Potsdam, Germany \\ and INFN, Sezione di Trieste, Via A. Valerio 2, I-34127 Trieste, Italy
}

(Received 29 November 2007; published 23 May 2008)

\begin{abstract}
We have studied extreme mass-ratio inspirals (EMRIs) in spacetimes containing a rotating black hole and a non-self-gravitating torus with a constant distribution of specific angular momentum. We have found that the dissipative effect of the hydrodynamic drag exerted by the torus on the satellite is much smaller than the corresponding one due to radiation reaction, for systems such as those generically expected in active galactic nuclei and at distances from the central supermassive black hole (SMBH) which can be probed with the Laser Interferometer Space Antenna (LISA). However, given the uncertainty on the parameters of these systems, namely, on the masses of the SMBH and of the torus, as well as on its size, there exist configurations in which the effect of the hydrodynamic drag on the orbital evolution can be comparable to the radiation reaction one in phases of the inspiral which are detectable by the Laser Interferometer Space Antenna. This is the case, for instance, for a $10^{6} M_{\odot} \mathrm{SMBH}$ surrounded by a corotating torus of comparable mass and with radius of $10^{3}-10^{4}$ gravitational radii, or for a $10^{5} M_{\odot} \mathrm{SMBH}$ surrounded by a corotating $10^{4} M_{\odot}$ torus with radius of $10^{5}$ gravitational radii. Should these conditions be met in astrophysical systems, EMRI-gravitational waves could provide a characteristic signature of the presence of the torus. In fact, while radiation reaction always increases the inclination of the orbit with respect to the equatorial plane (i.e., orbits evolve towards the equatorial retrograde configuration), the hydrodynamic drag from a torus corotating with the SMBH always decreases it (i.e., orbits evolve towards the equatorial prograde configuration). However, even when initially dominating over radiation reaction, the influence of the hydrodynamic drag decays very rapidly as the satellite moves into the very strong-field region of the SMBH (i.e., $p \lessgtr 5 M$ ), thus allowing one to use pure-Kerr templates for the last part of the inspiral. Although our results have been obtained for a specific class of tori, we argue that they will be qualitatively valid also for more generic distributions of the specific angular momentum.
\end{abstract}

DOI: 10.1103/PhysRevD.77.104027

\section{INTRODUCTION}

One of the most exciting prospects opened up by the scheduled launch of the space-based gravitational-wave detector known as the Laser Interferometer Space Antenna (LISA) [1] will be the possibility of mapping accurately the spacetime of the supermassive black holes (SMBHs) which are believed to reside in the center of galaxies [2]. Among the best candidate sources for this detector there are extreme mass-ratio inspirals (EMRIs), i.e. stellar-mass black holes $\left(m \approx 1-10 M_{\odot}\right)$ or compact objects orbiting around the SMBH and slowly inspiraling due to the loss of energy and angular momentum via gravitational waves (radiation reaction). In order for the signal to fall within the sensitivity band of LISA, the SMBH must have a mass $M \approx 10^{5}-10^{7} M_{\odot}$, i.e., the low end of the SMBH mass function.

It is currently expected that a number of such events ranging from tens to perhaps 1000 could be measured every year [3], but since they will have small signal-tonoise ratios, their detection and subsequent parameter extraction will require the use of matched-filtering tech-

\footnotetext{
*barausse@ sissa.it
}

PACS numbers: 04.30.-w, 04.70.-s, 98.35.Jk, 98.62.Js

niques. These basically consist of cross correlating the incoming gravitational-wave signal with a bank of theoretical templates representing the expected signal as a function of the parameters of the source.

This will not only allow one to detect the source, but also to extract its properties. For instance, the accurate modeling of the motion of a satellite in a Kerr spacetime will allow one to measure the spin and the mass of the SMBH. Although producing these pure-Kerr templates has proved to be a formidable task, particularly because of the difficulty of treating rigorously the effect of radiation reaction (see Ref. [4] for a detailed review), considerable effort has gone into trying to include the effects of a deviation from the Kerr geometry. These attempts are motivated by the fact that possible "exotic" alternatives to SMBHs have been proposed (e.g., boson stars [5], fermion balls, [6] and gravastars [7]), although the presence of these objects would require to modify radically the mechanism with which galaxies are expected to form. On the other hand, non-pure-Kerr templates might allow one to really map the spacetimes of SMBHs and to test experimentally the Kerr solution.

Different approaches to this problem have been considered in the literature. EMRIs in a spacetime having arbi- 
trary gravitational multipoles should be considered in order to maintain full generality [8], but this method does not work very well in practice and would only apply to vacuum spacetimes. For this reason, alternative approaches have been proposed and range from EMRIs around nonrotating boson stars [9], to EMRIs in bumpy black hole spacetimes [10] (i.e., spacetimes which are almost Schwarzschild and require naked singularities or exotic matter), or in quasiKerr spacetimes [11] (i.e., spacetimes consisting of Kerr plus a small quadrupole moment).

Interestingly, none of these methods is suitable for taking into account the effect of the matter which is certainly present in galactic centers. SMBHs can indeed be surrounded by stellar disks (as in the case of the Galactic center [12]) or as in the case of active galactic nuclei (AGNs) [13], in which we are most interested, by accretion disks of gas and dust which can be even as massive as the SMBH [14]. While the gravitational attraction of a disk can have important effects on EMRIs if this disk is very massive and close to the SMBH [15], an astrophysically realistic accretion disk can influence an EMRI only if the satellite crosses it, thus experiencing a "hydrodynamic" drag force.

This drag consists of two parts. The first one is due to the accretion of matter onto the satellite black hole (this was studied analytically by Bondi and Hoyle [16] and subsequently confirmed through numerical calculations [1719]). This transfers energy and momentum from the disk to the satellite, giving rise to a short-range interaction. The second one is instead due the gravitational deflection of the material which is not accreted, which is therefore far from the satellite, but which can nevertheless transfer momentum to it. This long-range interaction can also be thought of as arising from the gravitational pull of the satellite by its own gravitationally induced wake (i.e., the density perturbations that the satellite excites, by gravitational interaction, in the medium), and is often referred to as "dynamical friction." This effect was first studied in a collisionless medium by Chandrasekar [20], but acts also for a satellite moving in a collisional fluid [17,21-25].

The effect of this disk-satellite interaction on EMRIs has been studied by different authors for a number of disk models. More specifically, in a series of papers Karas, Subr and Vokrouhlicky considered the interaction between stellar satellites and thin disks [26-28]. In Ref. [28], in particular, Subr and Karas found that the effect of the stardisk interaction on EMRIs dominates over radiation reaction for thin disks, both for nonequatorial orbits crossing the disk only twice per revolution and for equatorial orbits embedded in the disk. The only exceptions to these conclusions come if the satellite is very compact (a neutron star or a black hole) or the disk has a low density (e.g., in the region close to the SMBH if the flow becomes advection-dominated). These results agree with those found by Narayan [29], who focused on advection- dominated accretions flows, which were believed to describe accretion onto "normal" galactic nuclei (i.e., ones much dimmer than AGNs). ${ }^{1}$ Overall, he found that for compact objects and white dwarfs the effect of the hydrodynamic drag is negligible with respect to radiation reaction, whereas it is not negligible for main sequence and giant stars. More recently, Levin [31] has proposed a scenario in which massive stars form in a thin accretion disk in an AGN, ultimately producing stellar-mass black holes embedded in the disk. The small black holes are then dragged towards the (nonrotating) $\mathrm{SMBH}$, but if this is accreting at a rate comparable to the Eddington limit, the drag from the accreting gas will not affect the final part of the inspiral (i.e., at radii smaller than 10 Schwarzschild radii) significantly. Finally, Chakrabarti [32] studied instead the orbital evolution of a satellite black hole on a circular equatorial orbit embedded in a disk with a nonKeplerian distribution of angular momentum, and found that the exchange of angular momentum between the disk and the satellite can lead to significant orbital modifications.

All of these studies have been carried out within a Newtonian or pseudo-Newtonian description of gravity (with the partial exception of Ref. [26], in which the orbits are Kerr geodesics, but the disk model and the hydrodynamic drag is not relativistic). In this paper, instead, we provide a first relativistic treatment for satellite black holes moving on generic orbits around a rotating SMBH surrounded by a thick disk (i.e., a torus). We consider the torus to have constant specific angular momentum and neglect its self-gravity (i.e., we consider the metric to be pure Kerr). Under these assumptions, an analytical solution exists for this system [33,34]. This configuration can be proved to be marginally stable with respect to axisymmetric perturbations [35] (i.e., if perturbed, such a torus can accrete onto the SMBH), and is expected to be a good approximation at least for the inner parts of the accretion flow $[33,34]$.

We have found that for a system composed of a SMBH with mass $M=10^{6} M_{\odot}$ and a torus with mass $M_{\mathrm{t}} \lesssim M$ and outer radius $r_{\text {out }}=10^{5} \mathrm{M}$, the effect of the hydrodynamic drag on the motion of the satellite black hole is much smaller than radiation reaction at those distances from the SMBH which can be probed with LISA (i.e., $\sim 10 M$ for $\left.M=10^{6} M_{\odot}\right)$. Although these values for $M, M_{\mathrm{t}}$ and $r_{\text {out }}$ are plausible for AGNs, an overall uncertainty is still present and has motivated an investigation also for different masses and sizes of the torus. In this way we have found that the effect of the torus can be important in the early part

\footnotetext{
${ }^{1}$ Accretion onto normal galactic nuclei is now believed to be better described by advection-dominated inflow-outflow solutions [30]. However, this is not expected to change significantly Narayan's results since advection-dominated inflow-outflow solutions (ADIOS), like advection-dominated accretions flows (ADAF), have very low densities in the vicinity of the SMBH.
} 
of the inspiral and that it could leave an observable imprint in the gravitational waveforms detected by LISA, if the radius of the torus is decreased to $r_{\text {out }}=10^{3}-10^{4} \mathrm{M}$ or, even for $r_{\text {out }}=10^{5} M$ and $M_{\mathrm{t}} \lesssim M$, if $M=10^{5} M_{\odot}$. In this latter case, in fact, LISA could detect an EMRI event at distances as large as $r \sim 45 M$ from the $\mathrm{SMBH}$, although the event needs to be sufficiently close to us because the amplitude of the gravitational-wave signal decreases as $M / r$.

In addition, if non-negligible, the effect of the hydrodynamic drag would have a distinctive signature on the waveforms. Radiation reaction, in fact, always increases the inclination of the orbit with respect to the equatorial plane (i.e., orbits evolve towards the equatorial retrograde configuration) [36]. The hydrodynamic drag from a torus corotating with the SMBH, on the other hand, always decreases this angle (i.e., orbits evolve towards the equatorial prograde configuration). Should such a behavior be observed in the data, it would provide a strong qualitative signature of the presence of the torus. However, it is important to point out that even for those configurations in which the hydrodynamic drag plays a major role, this is restricted to the initial part of the inspiral detectable by LISA, whereas its effect rapidly vanishes in the very strong-field region of the SMBH (i.e., $p \lesssim 5 M$ ). As a result, the pure-Kerr templates would provide a faithful description of the last part of the inspiral even in these cases.

The rest of the paper is organized as follows. In Sec. II we review the equilibrium solutions that we used for the orbiting torus. In Sec. III A we present the equations governing the interaction between the satellite black hole and the torus, while in Sec. III B we apply the adiabatic approximation to the hydrodynamic drag. Results are then discussed in Sec. IVA for equatorial circular orbits and in Sec. IV B for generic (inclined and eccentric) orbits. Finally, the conclusions are drawn in Sec. V. Throughout this paper we use units in which $G=c=1$.

\section{MODELING THE TORUS}

The properties of non-self-gravitating, stationary, axisymmetric and plane-symmetric toroidal fluid configurations in Kerr spacetimes are well known in astrophysics but are less well known within the community working on EMRIs. Because of this, in this section we briefly review the basic facts, referring the interested reader to Refs. [33,34,37-39] for additional information.

Let us consider a perfect fluid with 4-velocity $\boldsymbol{u}^{\text {fluid, }}$, which is described by the stress-energy tensor

$$
\begin{aligned}
T^{\mu \nu} & =(\rho+p) u_{\text {fluid }}^{\mu} u_{\text {fluid }}^{\nu}+p g^{\mu \nu} \\
& =\rho_{0} h u_{\text {fluid }}^{\mu} u_{\text {fluid }}^{\nu}+p g^{\mu \nu},
\end{aligned}
$$

where $p, \rho_{0}, \rho$, and $h \equiv(p+\rho) / \rho_{0}$ are the pressure, restmass density, energy density, and specific enthalpy of the fluid. In what follows we will model the fluid with a polytropic equation of state $p=\kappa \rho_{0}^{\Gamma}=\rho_{0} \varepsilon(\Gamma-1)$, where $\varepsilon=\rho / \rho_{0}-1$ is the internal energy per unit restmass, and $\kappa$ and $\Gamma$ are the polytropic constant and index, respectively. Because we are neglecting the self-gravity of the fluid, we can also consider $\boldsymbol{g}$ as given by the Kerr metric in Boyer-Lindquist coordinates, which reads [40]

$$
\begin{aligned}
d s^{2}= & -\left(1-\frac{2 M r}{\Sigma}\right) d t^{2}+\frac{\Sigma}{\Delta} d r^{2}+\Sigma d \theta^{2} \\
& +\left(r^{2}+a^{2}+\frac{2 M a^{2} r}{\Sigma} \sin ^{2} \theta\right) \sin ^{2} \theta d \phi^{2} \\
& -\frac{4 M a r}{\Sigma} \sin ^{2} \theta d t d \phi,
\end{aligned}
$$

where

$$
\Sigma \equiv r^{2}+a^{2} \cos ^{2} \theta, \quad \Delta \equiv r^{2}-2 M r+a^{2} .
$$

The fluid is assumed to be in circular nongeodesic motion with 4-velocity

$$
\begin{aligned}
\boldsymbol{u}^{\text {fluid }} & =A(r, \theta)\left[\frac{\partial}{\partial t}+\Omega(r, \theta) \frac{\partial}{\partial \phi}\right] \\
& =U(r, \theta)[-d t+\ell(r, \theta) d \phi],
\end{aligned}
$$

where the second equals sign underlines that the vector and the 1 -form are each the dual of the other. Here, $\Omega \equiv$ $u_{\text {fluid }}^{\phi} / u_{\text {fluid }}^{t}$ is the angular velocity, $A \equiv u_{\text {fluid }}^{t}$ is called the redshift factor, $U \equiv-u_{t}^{\text {fluid }}$ is the energy per unit mass as measured at infinity, and $\ell \equiv-u_{\phi}^{\text {fluid }} / u_{t}^{\text {fluid }}$ is the specific angular momentum as measured at infinity (i.e., the angular momentum per unit energy as measured at infinity). Note that $\ell$ is conserved for stationary axisymmetric flows, as can be easily shown using Euler's equation. The specific angular momentum and the angular velocity are trivially related by

$$
\Omega=-\frac{g_{t \phi}+g_{t t} \ell}{g_{\phi \phi}+g_{t \phi} \ell}, \quad \ell=-\frac{g_{t \phi}+g_{\phi \phi} \Omega}{g_{t t}+g_{t \phi} \Omega},
$$

while the normalization condition $\boldsymbol{u}^{\text {fluid }} \cdot \boldsymbol{u}^{\text {fluid }}=-1$ gives

$$
\begin{gathered}
U=\sqrt{\frac{\varpi^{2}}{g_{t t} \ell^{2}+2 g_{t \phi} \ell+g_{\phi \phi}}}, \\
A=\sqrt{\frac{-1}{g_{t t}+2 g_{t \phi} \Omega+g_{\phi \phi} \Omega^{2}}}, \\
A U=\frac{1}{1-\Omega \ell},
\end{gathered}
$$

where $\varpi^{2}=g_{t \phi}^{2}-g_{t t} g_{\phi \phi}=\Delta \sin ^{2} \theta$. Note that in this paper we will always consider $\ell>0$ (torus rotating in the positive $\phi$ direction), while we will allow the spin parameter $a$ of the black hole to be either positive (black 
hole corotating with the torus) or negative (black hole counterrotating with respect to the torus).

To calculate the structure of the torus, we need to use Euler's equation, which in its general form reads

$$
a_{\text {fluid }}^{\mu}=-\frac{\left(g^{\mu \nu}+u_{\text {fluid }}^{\mu} u_{\text {fluid }}^{\nu}\right) \partial_{\nu} p}{p+\rho},
$$

where $a_{\text {fluid }}^{\mu}$ is the 4-acceleration of the fluid. In particular, if the pressure is assumed to depend only on $r$ and $\theta$ and if the equation of state is barotropic [i.e., if $\rho=\rho(p)],{ }^{2}$ from Eq. (9) one easily gets that the 4-acceleration can be expressed as the gradient of a scalar potential $W(p)$ :

$$
a_{\mu}^{\text {fluid }}=\partial_{\mu} W, \quad W(p)=-\int^{p} \frac{d p^{\prime}}{p^{\prime}+\rho\left(p^{\prime}\right)} .
$$

On the other hand, from the definition of 4-acceleration $\left(a_{\text {fluid }}^{\mu}=u_{\text {fluid }}^{\nu} \nabla_{\nu} u_{\text {fluid }}^{\mu}\right)$, Eqs. (4), (7), and (8), and the Killing equation $\nabla_{(\mu} \xi_{\nu)}=0$ for $\xi=\partial / \partial t$ and $\xi=$ $\partial / \partial \phi$, one easily gets

$$
a_{\mu}^{\text {fluid }}=\partial_{\mu} W=-\frac{\partial_{\mu} p}{p+\rho}=\partial_{\mu} \ln U-\frac{\Omega}{1-\Omega \ell} \partial_{\mu} \ell .
$$

In particular, taking the derivative of this equation, antisymmetrizing and using the trivial fact that $\partial_{[\mu \nu]} W=$ $\partial_{[\mu \nu]} \ell=\partial_{[\mu \nu]} U=0$, we obtain that $\partial_{[\mu} \Omega \partial_{\nu]} \ell=0$. This implies $\nabla \Omega \propto \nabla \ell$ and thus that $\ell$ and $\Omega$ have the same contour levels [i.e., $\Omega=\Omega(\ell)$ ]. Using this fact, we can then write Eq. (11) in an integral form:

$$
\begin{aligned}
W-W_{\text {out }} & =-\int_{0}^{p} \frac{d p^{\prime}}{p^{\prime}+\rho\left(p^{\prime}\right)} \\
& =\ln U-\ln U_{\text {out }}-\int_{\ell_{\text {out }}}^{\ell} \frac{\Omega\left(\ell^{\prime}\right) d \ell^{\prime}}{1-\Omega\left(\ell^{\prime}\right) \ell^{\prime}},
\end{aligned}
$$

where $W_{\text {out }}$ and $\ell_{\text {out }}$ are the potential and specific angular momentum at the outer edge of the torus. ${ }^{3}$

In the case of a torus with constant specific angular momentum [i.e., $\ell(r, \theta)=$ const], Eq. (12) provides an analytical solution, because once $\ell$ has been fixed the integral on the right-hand side is zero and Eq. (6) gives an analytical expression for $U$ :

$$
W-W_{\text {out }}=-\int_{0}^{p} \frac{d p^{\prime}}{p^{\prime}+\rho\left(p^{\prime}\right)}=\ln U-\ln U_{\text {out }} .
$$

Note that if one requires that $W \rightarrow 0$ when $r \rightarrow+\infty$ (i.e., $W=0$ for an equipotential surface closing at infinity), this equation gives $W=\ln U: W>0$ then corresponds to open equipotential surfaces, while $W<0$ corresponds to closed equipotential surfaces. Interestingly, the potential well can

\footnotetext{
${ }^{2}$ This is of course the case for a polytropic equation of state, because $\rho=p /(\Gamma-1)+(p / \kappa)^{1 / \Gamma}$.

${ }^{3}$ Of course, $W_{\text {out }}$ and $\ell_{\text {out }}$ can be replaced by the values of $W$ and $\ell$ at the inner edge of the torus if this is present.
}

present a minimum and a saddle point. Because of the plane symmetry, these points are located in the equatorial plane, thus corresponding to local extremes of $W(r, \theta=$ $\pi / 2$ ), and mark two important positions, respectively, the center of the torus (i.e., the point where the density reaches its maximum) and its cusp (i.e., the mass-shedding point). Noticeably, these points are located at the radii where the specific angular momentum of the torus $\ell$ coincides with that of the geodesic circular equatorial orbit (the "Keplerian" orbit) corotating with the torus,

$$
\ell_{K}(r, a)=\frac{r^{2}-2 a \sqrt{M r}+a^{2}}{(r-2 M) \sqrt{r / M}+a} .
$$

This immediately follows from the fact that at the extremes of the function $W$ one has $\partial_{\mu} W=0$, which leads, through Eq. (10), to $a_{\text {fluid }}^{\mu}=0$ (in other words, at the center and at the cusp the pressure gradients are zero and only gravitational forces act).

In this paper we will indeed consider constant $\ell$ tori. A detailed classification of these models depending upon the values of $\ell$ and of $W_{\text {out }}$ can be found in Refs. [33,34,37]. Here we simply recall that in order to have a closed equipotential surface with a cusp, one needs to have a value of $\ell$ between the specific angular momenta $\ell_{\mathrm{ms}}$ and $\ell_{\mathrm{mb}}$ of the marginally stable and marginally bound equatorial geodesic (i.e., Keplerian) orbits corotating with the torus. This can be easily understood by noting, from Eq. (13), that the potential $W(r, \theta=\pi / 2)$ is simply the effective potential describing the equatorial motion of a test particle around a Kerr black hole. As such, $\ell_{\mathrm{ms}}$ and $\ell_{\mathrm{mb}}$ can be calculated easily using Eq. (14) and the formulas for the radii of the marginally stable and marginally bound circular equatorial orbits in Kerr rotating in the positive $\phi$ direction (i.e., corotating with the torus):

$$
\begin{gathered}
\ell_{\mathrm{ms}}=\ell_{K}\left(r_{\mathrm{ms}}\right), \quad \ell_{\mathrm{mb}}=\ell_{K}\left(r_{\mathrm{mb}}\right) \\
r_{\mathrm{ms}} / M=3+Z_{2}-\operatorname{sgn}(\tilde{a}) \sqrt{\left(3-Z_{1}\right)\left(3+Z_{1}+2 Z_{2}\right)} \\
r_{\mathrm{mb}} / M=2-\tilde{a}+2 \sqrt{1-\tilde{a}} \\
Z_{1}=1+\left(1-\tilde{a}^{2}\right)^{1 / 3}\left[(1+\tilde{a})^{1 / 3}+(1-\tilde{a})^{1 / 3}\right] \\
Z_{2}=\sqrt{3 \tilde{a}^{2}+Z_{1}^{2}}
\end{gathered}
$$

where $\tilde{a}=a / M$.

In order to pick up a particular solution having both an inner and an outer radius, one needs also to choose a negative value for the "potential barrier" at the inner edge of the torus,

$$
\Delta W=W_{\text {in }}-W_{\text {cusp }}=W_{\text {out }}-W_{\text {cusp }} \leq 0 .
$$

If $\Delta W<0$, the inner radius of the torus is larger than the 
radius at which the cusp occurs ( $\left.r_{\text {in }}>r_{\text {cusp }}\right)$, while if the potential barrier $\Delta W$ reduces to zero, the torus exactly fills its outermost closed equipotential surface and $r_{\text {in }}=$ $r_{\text {cusp }} \leq r_{\mathrm{ms}}$. Note that because of the considerations that we have made above about the value of $\ell$, for constant $\ell$ tori we have $r_{\text {cusp }} \geq r_{\text {mb }}$ (with $r_{\text {cusp }}=r_{\text {mb }}$ only if $\ell=\ell_{\mathrm{mb}}$ ) and $r_{\text {center }} \geq r_{\mathrm{ms}}$ (with $r_{\text {center }}=r_{\mathrm{ms}}$ only if $\ell=\ell_{\mathrm{ms}}$ ). If instead $\Delta W>0$, the fluid overflows the outermost closed equipotential surface and mass transfer is possible at the cusp: for a polytropic equation of state, the accretion rate can be shown to be $\dot{M} \propto \Delta W^{\Gamma /(\Gamma-1)}$.

The integral Euler equation for constant- $\ell$ tori [Eq. (13)] further simplifies if the equation of state is polytropic, because in this case

$$
\int_{0}^{p} \frac{d p^{\prime}}{p^{\prime}+\rho\left(p^{\prime}\right)}=\ln \frac{h}{h_{\text {out }}}
$$

where $h_{\text {out }}$ is the specific enthalpy at the outer edge of the torus. Since for a polytropic equation of state the enthalpy is given by

$$
h=1+\frac{\Gamma}{\Gamma-1} \kappa \rho_{0}^{\Gamma-1},
$$

it is clear that $h_{\text {out }}=1$ (because $p=\rho_{0}=0$ at the outer edge of the torus), and Eqs. (13) and (21) give

$$
\rho_{0}(r, \theta)=\left\{\frac{\Gamma-1}{\Gamma} \frac{\left[e^{W_{\text {out }}-W(r, \theta)}-1\right]}{\kappa}\right\}^{1 /(\Gamma-1)} .
$$

Once the rest-mass distribution is known, the total restmass of the torus is given by

$$
M_{t, 0}=\int \rho_{0} \sqrt{-g} u^{t} d^{3} x,
$$

where $\sqrt{-g}=\Sigma \sin \theta$ and $d^{3} x=d r d \theta d \phi$ is the coordinate 3 -volume element, while the mass-energy reads

$$
\begin{aligned}
M_{\mathrm{t}}= & \int\left(T_{r}^{r}+T_{\phi}^{\phi}+T_{\theta}^{\theta}-T_{t}^{t}\right) \sqrt{-g} d^{3} x \\
= & 2 \pi \int_{\rho_{0}>0}\left(\frac{g_{\phi \phi}-g_{t \ell} \ell^{2}}{g_{\phi \phi}+2 g_{t \phi} \ell+g_{t t} \ell^{2}} \rho_{0} h+2 P\right) \\
& \times\left(r^{2}+a^{2} \cos ^{2} \theta\right) \sin \theta d r d \theta .
\end{aligned}
$$

Clearly, the smaller the ratio between the mass of the torus and that of the SMBH, the better the approximation of neglecting the self-gravity of the torus.

\section{MODELING THE ORBITAL MOTION}

This section is dedicated to the discussion of the hydrodynamic drag on the satellite black hole. Although the two aspects are closely interrelated, we first discuss the equations governing the interaction between the satellite black hole and the torus and then describe their use in the calculation of the changes of the orbital parameters within the adiabatic approximation.

\section{A. Hydrodynamic drag}

As already mentioned in Sec. I, the hydrodynamic drag acting on the satellite black hole can be written as the sum of a short-range part, due to accretion, and a long-range part, due to the deflection of the matter which is not accreted or, equivalently, to the gravitational interaction of the satellite with the density perturbations gravitationally induced by its own presence:

$$
\frac{d p_{\mathrm{sat}}^{\mu}}{d \tau}=\left.\frac{d p^{\mu}}{d \tau}\right|_{\mathrm{accr}}+\left.\frac{d p^{\mu}}{d \tau}\right|_{\mathrm{defl}},
$$

where $\tau$ is the proper time of the satellite.

Accretion onto a moving black hole was studied analytically in a Newtonian framework by Bondi and Hoyle [16], who found the rest-mass accretion rate to be

$$
\frac{d m_{0}}{d \tau}=\frac{4 \pi \lambda m^{2} \rho_{0}}{\left(v^{2}+v_{s}^{2}\right)^{3 / 2}}
$$

where $m$ is the mass of the black hole, $v$ and $v_{s}$ are, respectively, the velocity of the black hole with respect to the fluid and the sound velocity, and $\lambda$ is a dimensionless constant of the order of unity, which for a fluid with polytropic equation of state and polytropic index $\Gamma$ has the value [41]

$$
\lambda=\left(\frac{1}{2}\right)^{(\Gamma+1) /[2(\Gamma-1)]}\left(\frac{5-3 \Gamma}{4}\right)^{-(5-3 \Gamma) /[2(\Gamma-1)]} .
$$

Subsequent numerical work [17-19] treated instead the problem of accretion in full general relativity, and showed that Eq. (27), with $\lambda$ given by Eq. (28), is correct provided that it is multiplied by a factor $\sim 5-25$ when $v$ and $v_{s}$ become relativistic (cf. Table 3 of Ref. [18]). However, because a fit for this correction factor is, to the best of our knowledge, not yet available and the published data are not sufficient for producing one, we use the Bondi accretion rate [Eqs. (27) and (28)], bearing in mind that it could slightly underestimate the drag at relativistic velocities $v$ and $v_{s}{ }^{4}$ Once the accretion rate is known, the short-range part of the drag reads [17]

$$
\left.\frac{d p^{\mu}}{d \tau}\right|_{\text {accr }}=h \frac{d m_{0}}{d \tau} u_{\text {fluid }}^{\mu},
$$

where we recall that $h$ is the specific enthalpy of the fluid. Note that this equation basically follows from the conservation of the total 4-momentum of the satellite and the fluid.

\footnotetext{
${ }^{4}$ As we will see in Sec. IV, $v$ and $v_{s}$ can become relativistic only for orbits counterrotating with respect to the torus and very close to the SMBH. For these orbits the dominant part of the hydrodynamic drag is the long-range one, and the relativistic correction factor to the Bondi accretion rate (which is roughly 510 for these orbits, as can be seen comparing the middle panel of Fig. 1 with Table 3 of Ref. [18]) does not change this conclusion.
} 
The long-range drag is instead more complicated. The gravitational interaction of a body with the density perturbations that it excites gravitationally in the surrounding medium was first studied by Chandrasekhar [20] in the case of a collisionless fluid, and is also known as "dynamical friction." Although less well recognized, dynamical friction acts also for a body moving in a collisional medium [17,21-25]. In particular, a satellite moving on a circular planar orbit (e.g., a circular orbit around a Schwarzschild black hole or a circular equatorial orbit around a Kerr black hole) experiences a drag in the tangential direction [17,21-23] and one in the radial direction [24]:

$$
\left.\frac{d p^{\mu}}{d \tau}\right|_{\text {defl }}=\left.\frac{d p}{d \tau}\right|_{\text {defl }} ^{\text {tang }} \sigma^{\mu}+\left.\frac{d p}{d \tau}\right|_{\text {defl }} ^{\text {rad }} \chi^{\mu},
$$

where $\boldsymbol{\sigma}$ is a unit spacelike vector orthogonal to $\boldsymbol{u}_{\text {sat }}$ and pointing in the direction of the motion of the fluid,

$$
\boldsymbol{\sigma}=\frac{\boldsymbol{u}_{\text {fluid }}-\gamma \boldsymbol{u}_{\mathrm{sat}}}{\sqrt{\gamma^{2}-1}}
$$

(the Lorentz factor $\gamma=-\boldsymbol{u}_{\text {fluid }} \cdot \boldsymbol{u}_{\text {sat }}$ encodes the relative motion of the satellite with respect to the fluid of the torus), and

$$
\boldsymbol{\chi}=-\frac{u_{r}^{\mathrm{sat}} \boldsymbol{u}_{\mathrm{sat}}-\sigma_{r} \boldsymbol{\sigma}+\partial / \partial r}{\left[g_{r r}-\left(u_{r}^{\mathrm{sat}}\right)^{2} /\left(\gamma^{2}-1\right)\right]^{1 / 2}},
$$

is a unit spacelike vector, orthogonal to both $\boldsymbol{u}_{\text {sat }}$ and $\boldsymbol{\sigma}$ and pointing in the radial direction. In particular, the tangential and radial drags are given by $[24,25]$

$$
\begin{gathered}
\left.\frac{d p}{d \tau}\right|_{\text {defl }} ^{\mathrm{tang}}=\frac{4 \pi(p+\rho) m^{2} \gamma^{2}\left(1+v^{2}\right)^{2}}{v^{2}} I_{\mathrm{tang}}, \\
\left.\frac{d p}{d \tau}\right|_{\mathrm{defl}} ^{\mathrm{rad}}=\frac{4 \pi(p+\rho) m^{2} \gamma^{3}\left(1+v^{2}\right)^{2}}{v^{2}} I_{\mathrm{rad}},
\end{gathered}
$$

where $I_{\text {tang }}$ and $I_{\text {rad }}$ are complicated integrals. Fits to the numerically computed steady-state ${ }^{5}$ values for these integrals are given in Ref. [24]:

$$
I_{\text {tang }}= \begin{cases}0.7706 \ln \left(\frac{1+\mathcal{M}}{1.0004-0.9185 \mathcal{M}}\right)-1.4703 \mathcal{M} & \text { for } \mathcal{M}<1.0 \\ \ln \left[330\left(r / r_{\min }\right)(\mathcal{M}-0.71)^{5.72} \mathcal{M}^{-9.58}\right] & \text { for } 1.0 \leq \mathcal{M}<4.4 \\ \ln \left[\left(r / r_{\min }\right) /(0.11 \mathcal{M}+1.65)\right] & \text { for } \mathcal{M} \geq 4.4\end{cases}
$$

and

$$
I_{\text {rad }}= \begin{cases}\mathcal{M}^{2} 10^{3.51 \mathcal{M}-4.22} & \text { for } \mathcal{M}<1.1 \\ 0.5 \ln \left[9.33 \mathcal{M}^{2}\left(\mathcal{M}^{2}-0.95\right)\right] & \text { for } 1.1 \leq \mathcal{M}<4.4 \\ 0.3 \mathcal{M}^{2} & \text { for } \mathcal{M} \geq 4.4\end{cases}
$$

where $r$ is the radius of the circular orbit, $r_{\min } \sim 2 m(1+$ $\left.v^{2}\right) / v^{2}$ is the capture impact parameter of the satellite black hole, while $\mathcal{M}=v / v_{s}$ is the Mach number.

These fits are valid for $r \gg r_{\min }$ and are accurate within $4 \%$ for $\mathcal{M}<4.4$ and within $16 \%$ for $\mathcal{M}>4$.4. However, the fit for $I_{\text {tang }}$ does not go to zero when $\mathcal{M}$ goes to zero, while $I_{\text {rad }}$ goes to zero only as $\mathcal{M}^{2}$ in this limit: these behaviors would give a nonzero radial drag and a diverging tangential drag for $v \rightarrow 0$ [cf. Eqs. (33) and (34)]. This is clearly a spurious behavior: dynamical friction must vanish for $v=0$, since in this case the pattern of the density perturbations is spherically symmetric around the body (as there is no preferred direction). However, as we will see in Sec. III B, the effect of the radial drag vanishes if one uses the adiabatic approximation (as it is usually done in EMRI studies [42-45]), and therefore this artifact of the fit (36) cannot cause any harm in our numerical code. This is instead not the case for the tangential drag: in order to eliminate its spurious divergence, we have approximated $I_{\text {tang }}$ with its straightline functional form at low Mach numbers. Since the dynamical friction drag for straightline subsonic motion is given by Eq. (33) with $I_{\text {tang }}=$
$1 / 2 \ln [(1+\mathcal{M}) /(1-\mathcal{M})]-\mathcal{M} \approx \mathcal{M}^{3} / 3+\mathcal{M}^{5} / 5$, we can assume that $I_{\text {tang }}$ is given, for $\mathcal{M}<0.1$, by

$$
I_{\text {tang }}=0.9563\left(\frac{\mathcal{M}^{3}}{3}+\frac{\mathcal{M}^{5}}{5}\right)
$$

where the factor 0.9563 is introduced to match the above fit at $\mathcal{M}=0.1$.

Note that although Eq. (30) is strictly valid only for circular planar motion (i.e., in the case of a Kerr spacetime, for circular equatorial orbits), we expect it to be a good approximation also for generic orbits around a Kerr black hole. Indeed, thanks to the choice of the unit vectors $\boldsymbol{\sigma}$ and $\boldsymbol{\chi}$, Eq. (30) gives a tangential drag parallel the direction of the flow and a drag in the radial direction perpendicular to the direction of the flow. Both of these components are expected to be present also for generic orbits. In particular, the tangential drag should be given approximately by

\footnotetext{
${ }^{5}$ Fortunately, the steady-state values for these integrals are reached over time scales which are comparable with either the sound crossing time $r / v_{s}, r$ being the radius of the circular orbit, or with the orbital period.
} 
Eqs. (33) and (37) if the radius $r$ appearing in Eq. (35) is replaced by the semilatus rectum $p$ of the orbit [see Eq. (52) for the definition of this quantity]. ${ }^{6}$ Although this prescription is not exact, the results of Ref. [24] suggest that the relevant length scale in the Coulomb logarithm appearing in the second and third lines of Eq. (35) should be one characterizing the orbit, rather than the size of the medium, as commonly assumed in most of the works on dynamical friction predating Refs. [22,24] (see the introduction of Ref. [24] and references therein for more details about this point). Of course, this length scale could be different from the semilatus rectum of the orbit, but different choices for it would have only a slight impact on the results because of the logarithmic dependence.

The extrapolation of the radial drag given by Eqs. (34) and (36) from circular planar to generic orbits is instead a bit more problematic, although one expects it to be a good approximation at least for orbits with small eccentricities and small inclinations with respect to the equatorial plane. At any rate, as we have mentioned earlier, in Sec. III B we will show that the effect of this radial drag on the orbital evolution averages to zero when adopting the adiabatic approximation. (Note that this agrees with Ref. [24], which found that the effect of the radial drag on the orbital evolution was subdominant with respect to that of the tangential drag.) Nevertheless, a nonzero effect may still be present in cases in which the adiabatic approximation is not valid (i.e. if the hydrodynamic drag acts on a time scale comparable to the orbital period), or possibly even in the adiabatic approximation if more rigorous expressions for the radial drag should be derived in the future.

The rate of change of the mass of the satellite with respect to the coordinate time $t$ follows immediately from $p_{\text {sat }}^{\mu} p_{\mu}^{\text {sat }}=-m^{2}$ : denoting the derivative with respect to $t$ with an overdot, we have

$$
\dot{m}=-\frac{u_{\mu}^{\mathrm{sat}}}{u_{\mathrm{sat}}^{t}} \frac{d p_{\mathrm{sat}}^{\mu}}{d \tau}=-\frac{u_{\mu}^{\mathrm{sat}}}{u_{\mathrm{sat}}^{t}} \frac{d p_{\mathrm{accr}}^{\mu}}{d \tau}=\frac{h \gamma}{u_{\mathrm{sat}}^{t}} \frac{d m_{0}}{d \tau} .
$$

It is well known [46] that Kerr geodesics can be labeled, up to initial conditions, by three constants of motion, the dimensionless energy $\tilde{E}$ and the angular momentum $\tilde{L}_{z}$ as measured by an observer at infinity,

$$
\tilde{E}=-u_{t}^{\mathrm{sat}}, \quad \tilde{L}_{z}=u_{\phi}^{\mathrm{sat}} / M,
$$

and the dimensionless Carter constant [46] $\tilde{Q}$,

\footnotetext{
${ }^{6}$ Note that the tangential drag given by Eqs. (33), (35), and (37) is approximately correct also for straightline motion, if $r$ replaced in Eq. (35) by $v t-t$ being the time for which the satellite has been active [22,25] - as long as $v t$ is smaller than the size of the medium, and by a cutoff length of order of the size of the medium at later times. To see this, compare Eqs. (35) and (37) to the functional form of $I_{\text {tang }}$ for straightline motion, which is $I_{\text {tang }}=1 / 2 \ln [(1+\mathcal{M}) /(1-\mathcal{M})]-\mathcal{M}$ for subsonic motion and $I_{\text {tang }}=1 / 2 \ln \left(1-1 / \mathcal{M}^{2}\right)+\ln \left(v t / r_{\min }\right)$ for supersonic motion $[22,25]$.
}

$$
\tilde{Q}=\left(\frac{u_{\theta}^{\mathrm{sat}}}{M}\right)^{2}+\tilde{a}^{2} \cos ^{2} \theta\left(1-\tilde{E}^{2}\right)+\cot ^{2} \theta \tilde{L}_{z}^{2}
$$

where $\tilde{a}=a / M$. We will now derive expression for the rates of change of these quantities.

To this purpose, let us first introduce the tetrad $\left(\boldsymbol{u}_{\mathrm{sat}}, \boldsymbol{e}_{1}=\boldsymbol{\sigma}, \boldsymbol{e}_{2}=\boldsymbol{\chi}, \boldsymbol{e}_{3}\right)$ based in the position of the satellite and write the change in the 4-velocity due to accretion and deflection of the flow as

$$
\delta u_{\mathrm{sat}}^{\mu}=\delta u_{\mathrm{sat}}^{(t)} u_{\mathrm{sat}}^{\mu}+\delta u_{\mathrm{sat}}^{(i)} e_{(i)}^{\mu},
$$

where $\delta u_{\text {sat }}^{(t)}$ and $\delta u_{\text {sat }}^{(i)}$ are the components with respect to the tetrad. In particular, perturbing $-\left(u_{\mathrm{sat}}^{(t)}\right)^{2}+\delta_{i j} u_{\mathrm{sat}}^{(i)} u_{\mathrm{sat}}^{(j)}=$ -1 to first order one easily gets $-u_{\text {sat }}^{(t)} \delta u_{\text {sat }}^{(t)}+$ $\delta_{i j} u_{\mathrm{sat}}^{(i)} \delta u_{\mathrm{sat}}^{(j)}=0$, and using then the fact that $u_{\mathrm{sat}}^{(i)}=0$ to zeroth order, one obtains $\delta u_{\text {sat }}^{(t)}=0$. Using now $\delta u_{\text {sat }}^{(i)}=$ $\delta p_{\text {sat }}^{(i)} / m, \boldsymbol{e}_{(i)} \cdot \boldsymbol{u}_{\text {sat }}=0$ and $\boldsymbol{e}_{(i)} \cdot \boldsymbol{e}_{(j)}=\delta_{i j}(i=1,2,3)$, Eq. (41) becomes

$$
\begin{aligned}
\delta u_{\mathrm{sat}}^{\mu} & =\left(\frac{\delta m_{0} h}{m} u_{\mathrm{fluid}}^{\nu} \sigma_{\nu}+\frac{\delta p_{\mathrm{defl}}^{\mathrm{tang}}}{m}\right) \sigma^{\mu}+\frac{\delta p_{\mathrm{defl}}^{\mathrm{rad}}}{m} \chi^{\mu} \\
& =\left(\frac{\delta m_{0} h}{m}+\frac{\delta p_{\mathrm{defl}}^{\mathrm{tang}}}{m \sqrt{\gamma^{2}-1}}\right)\left(u_{\mathrm{fluid}}^{\mu}-\gamma u_{\mathrm{sat}}^{\mu}\right)+\frac{\delta p_{\mathrm{defl}}^{\mathrm{rd}}}{m} \chi^{\mu} .
\end{aligned}
$$

Using now Eqs. (4), (39), and (42), we immediately obtain

$$
\begin{aligned}
& \frac{\dot{\tilde{E}}}{\tilde{E}}=\left(\frac{\dot{m}_{0} h}{m}+\frac{\dot{p}_{\mathrm{def}}^{\mathrm{tang}}}{m \sqrt{\gamma^{2}-1}}\right)\left(\frac{U}{\tilde{E}}-\gamma\right)-\frac{\dot{p}_{\mathrm{defl}}^{\mathrm{rad}}}{m \tilde{E}} \chi_{t}, \\
& \frac{\dot{\tilde{L}}_{z}}{\tilde{L}_{z}}=\left(\frac{\dot{m}_{0} h}{m}+\frac{\dot{p}_{\mathrm{defl}}^{\mathrm{tang}}}{m \sqrt{\gamma^{2}-1}}\right)\left(\frac{\ell U}{M \tilde{L}_{z}}-\gamma\right)+\frac{\dot{p}_{\mathrm{delf}}^{\mathrm{rad}}}{m M \tilde{L}_{z}} \chi_{\phi} .
\end{aligned}
$$

In order to calculate instead the rate of change of the dimensionless Carter constant $\tilde{Q}$, let us note that from Eq. (42) it follows that the variation of $u_{\theta}$ in a short time interval $\delta t$ due to accretion and deflection of the flow is

$$
\delta u_{\theta}^{\mathrm{sat}}=\left[-\gamma\left(\frac{\dot{m}_{0} h}{m}+\frac{\dot{p}_{\mathrm{defl}}^{\mathrm{tang}}}{m \sqrt{\gamma^{2}-1}}\right) u_{\theta}^{\mathrm{sat}}+\frac{\dot{p}_{\mathrm{defl}}^{\mathrm{rad}}}{m} \chi_{\theta}\right] \delta t .
$$

We can then write $\dot{u}_{\theta}^{\text {sat }}$ as the sum of a term coming from the gravitational evolution (i.e., the geodesic equation) and one coming from collisions with the surrounding gas:

$$
\dot{u}_{\theta}^{\mathrm{sat}}=\Gamma_{\theta \nu}^{\mu} u_{\mu}^{\mathrm{sat}} \dot{\mathrm{s}}_{\mathrm{sat}}^{\nu}-\gamma\left(\frac{\dot{m}_{0} h}{m}+\frac{\dot{p}_{\mathrm{defl}}^{\mathrm{tang}}}{m \sqrt{\gamma^{2}-1}}\right) u_{\theta}^{\mathrm{sat}}+\frac{\dot{p}_{\mathrm{defl}}^{\mathrm{rad}}}{m} \chi_{\theta} .
$$

The evolution of $\tilde{Q}$ therefore follows from Eq. (40): 


$$
\begin{aligned}
\dot{\tilde{Q}}= & \frac{\partial \tilde{Q}}{\partial \theta} \dot{\theta}_{\mathrm{sat}}+\frac{\partial \tilde{Q}}{\partial u_{\theta}^{\mathrm{sat}}} \Gamma_{\theta \nu}^{\mu} u_{\mu}^{\mathrm{sat}} \dot{x}_{\mathrm{sat}}^{\nu}+\frac{\partial \tilde{Q}}{\partial \tilde{E}} \dot{\tilde{E}}+\frac{\partial \tilde{Q}}{\partial \tilde{L}_{z}} \dot{\tilde{L}}_{z} \\
& -\frac{\partial \tilde{Q}}{\partial u_{\theta}^{\mathrm{sat}}} \gamma\left(\frac{\dot{m}_{0} h}{m}+\frac{\dot{p}_{\mathrm{defl}}^{\mathrm{tang}}}{m \sqrt{\gamma^{2}-1}}\right) u_{\theta}^{\mathrm{sat}}+\frac{\partial \tilde{Q}}{\partial u_{\theta}^{\mathrm{sat}}} \frac{\dot{p}_{\mathrm{defl}}^{\mathrm{rad}}}{m} \chi_{\theta} \\
= & \frac{\partial \tilde{Q}}{\partial \tilde{E}} \dot{\tilde{E}}+\frac{\partial \tilde{Q}}{\partial \tilde{L}_{z}} \dot{\tilde{L}}_{z}-\frac{\partial \tilde{Q}}{\partial u_{\theta}^{\mathrm{sat}}} \gamma\left(\frac{\dot{m}_{0} h}{m}+\frac{\dot{p}_{\mathrm{defl}}^{\mathrm{tang}}}{m \sqrt{\gamma^{2}-1}}\right) u_{\theta}^{\mathrm{sat}} \\
& +\frac{\partial \tilde{Q}}{\partial u_{\theta}^{\mathrm{sat}}} \frac{\dot{p}_{\text {defl }}^{\mathrm{rad}}}{m} \chi_{\theta},
\end{aligned}
$$

where the partial derivatives are meant to be calculated with Eq. (40). Note that the first and the second term of the first line cancel out because $\tilde{Q}$ is conserved for geodesic motion.

A useful alternative form for the evolution rate of $\tilde{Q}$ can be obtained by rewriting Eq. (40) using the normalization condition $\boldsymbol{u}^{\text {sat }} \cdot \boldsymbol{u}^{\text {sat }}=-1$ :

$$
\begin{aligned}
\tilde{Q}= & \tilde{\Delta}^{-1}\left[\tilde{E}\left(\tilde{r}^{2}+\tilde{a}^{2}\right)-\tilde{a} \tilde{L}\right]^{2} \\
& -(\tilde{L}-\tilde{a} \tilde{E})^{2}-\tilde{r}^{2}-\tilde{\Delta}\left(u_{r}^{\text {sat }}\right)^{2},
\end{aligned}
$$

where $\tilde{r}=r / M$ and $\tilde{\Delta}=\Delta / M^{2}$. Proceeding as above and, in particular, using the fact that

$$
\dot{u}_{r}^{\mathrm{sat}}=\Gamma_{r \nu}^{\mu} u_{\mu}^{\mathrm{sat}} \dot{x}_{\mathrm{sat}}^{\nu}-\gamma\left(\frac{\dot{m}_{0} h}{m}+\frac{\dot{p}_{\mathrm{defl}}^{\mathrm{tang}}}{m \sqrt{\gamma^{2}-1}}\right) u_{r}^{\mathrm{sat}}+\frac{\dot{p}_{\mathrm{defl}}^{\mathrm{rad}}}{m} \chi_{r}
$$

[from Eqs. (4) and (42)], one easily gets

$$
\begin{aligned}
\dot{\tilde{Q}}= & \frac{\partial \tilde{Q}}{\partial r} \dot{r}_{\text {sat }}+\frac{\partial \tilde{Q}}{\partial u_{r}^{\text {sat }}} \Gamma_{r \nu}^{\mu} u_{\mu}^{\text {sat }} \dot{x}_{\text {sat }}^{\nu}+\frac{\partial \tilde{Q}}{\partial \tilde{E}} \dot{\tilde{E}}+\frac{\partial \tilde{Q}}{\partial \tilde{L}_{z}} \dot{\tilde{L}}_{z} \\
& -\frac{\partial \tilde{Q}}{\partial u_{r}^{\text {sat }}} \gamma\left(\frac{\dot{m}_{0} h}{m}+\frac{\dot{p}_{\text {defl }}^{\text {tang }}}{m \sqrt{\gamma^{2}-1}}\right) u_{r}^{\text {sat }}+\frac{\partial \tilde{Q}}{\partial u_{r}^{\text {sat }}} \frac{\dot{p}_{\text {defl }}^{\text {rad }}}{m} \chi_{r} \\
= & \frac{\partial \tilde{Q}}{\partial \tilde{E}} \dot{\tilde{E}}+\frac{\partial \tilde{Q}}{\partial \tilde{L}_{z}} \dot{\tilde{L}}_{z}-\frac{\partial \tilde{Q}}{\partial u_{r}^{\text {sat }}} \gamma\left(\frac{\dot{m}_{0} h}{m}+\frac{\dot{p}_{\text {defl }}^{\text {tang }}}{m \sqrt{\gamma^{2}-1}}\right) u_{r}^{\text {sat }} \\
& +\frac{\partial \tilde{Q}}{\partial u_{r}^{\text {sat }}} \frac{\dot{p}_{\text {defl }}^{\text {rad }}}{m} \chi_{r},
\end{aligned}
$$

where the partial derivatives are now calculated with Eq. (48). Note that for circular orbits Eq. (50) becomes

$$
\dot{\tilde{Q}}=\frac{\partial \tilde{Q}}{\partial \tilde{E}} \dot{\tilde{E}}+\frac{\partial \tilde{Q}}{\partial \tilde{L}_{z}} \dot{\tilde{L}}_{z}
$$

[use Eq. (48) and the fact that $u_{r}^{\text {sat }}=0$ for circular orbits]. This condition ensures ${ }^{7}$ that circular orbits keep circular

\footnotetext{
${ }^{7}$ Note, in particular, that the proof presented in Ref. [47], which was concerned mainly with radiation reaction, applies also to the case of the hydrodynamic drag. Note also that the resonance condition which was found in Ref. [47] as the only possible case that could give rise to a noncircular evolution for an initially circular orbit is never satisfied in a Kerr spacetime [48].
}

under the hydrodynamic drag and in the adiabatic approximation, as it happens for radiation reaction.

Finally, let us note that the rates of change of $\tilde{E}, \tilde{L}_{z}$ and $\tilde{Q}$ [Eqs. (43), (44), (47), and (50)] go smoothly to zero as the velocity of the satellite relative to the fluid goes to zero. This is easy to check using the fact that, when $v$ approaches zero, $\dot{p}_{\text {defl }}^{\text {tang }}=\mathcal{O}(v)$ [cf. Eqs. (33) and (37)], $\dot{p}_{\text {defl }}^{\text {rad }} \rightarrow 0$, $\gamma^{2}-1=\mathcal{O}\left(v^{2}\right), u_{r}=\mathcal{O}(v), u_{\theta}=\mathcal{O}(v), \ell U-M \tilde{L}_{z}=$ $\mathcal{O}(v)$ and $U-\tilde{E}=\mathcal{O}\left(v^{2}\right)$, and using the fact that $\boldsymbol{\chi}$ keeps finite in this limit [in particular, from Eqs. (31) and (32) it follows $\chi_{t}=\mathcal{O}(v), \chi_{\phi}=\mathcal{O}(1), \chi_{\theta}=\mathcal{O}(1)$, and $\chi_{r}=$ $\mathcal{O}(1)]$. Note that this is indeed the result that one would expect. First of all, a body comoving with the fluid clearly does not experience any dynamical friction and the only active mechanism is accretion. The body then accretes mass and consequently energy and angular momentum (because the fluid carries a specific energy and a specific angular momentum). However, the dimensionless constants of motion $\tilde{E}, \tilde{L}_{z}$, and $\tilde{Q}$ entering the geodesic equation cannot change because of the weak equivalence principle. Pictorially, one may think of a satellite comoving with a gaseous medium. Consider a sphere centered in the satellite, with radius small enough to ensure that the gas contained in the sphere has approximately the same velocity as the satellite. Suppose now that all the gas in this sphere is accreted by the satellite. The velocity of the satellite will clearly be unaffected, because of the conservation of momentum: for the weak equivalence principle this is enough to ensure that the orbit of the satellite will be unaffected, in spite of its increased mass.

\section{B. Adiabatic approximation}

At the heart of our approach is the calculation of the changes of the orbital parameters experienced by Kerr geodesics as a result of the hydrodynamic drag, and their comparison with the corresponding changes introduced by radiation reaction. To this purpose, let us recall that up to initial conditions Kerr geodesics can be labeled by a set of three parameters, the semilatus rectum $p$, the eccentricity $e$, and the inclination angle $\theta_{\text {inc }}$. These are just a remapping of the energy, angular momentum, and Carter constant introduced in Sec. III A, and are defined as [49]

$$
p=\frac{2 r_{\mathrm{a}} r_{\mathrm{p}}}{r_{\mathrm{a}}+r_{\mathrm{p}}}, \quad e=\frac{r_{\mathrm{a}}-r_{\mathrm{p}}}{r_{\mathrm{a}}+r_{\mathrm{p}}}, \quad \theta_{\mathrm{inc}}=\frac{\pi}{2}-D \theta_{\text {min }},
$$

where $r_{\mathrm{a}}$ and $r_{\mathrm{p}}$ are the apastron and periastron coordinate radii, $\theta_{\min }$ is the minimum polar angle $\theta$ reached during the orbital motion and $D=1$ for orbits corotating with the SMBH whereas $D=-1$ for orbits counterrotating with respect to it. Note that in the weak-field limit $p$ and $e$ correspond exactly to the semilatus rectum and eccentricity used to describe orbits in Newtonian gravity, and that $\theta_{\text {inc }}$ goes from $\theta_{\text {inc }}=0$ for equatorial orbits corotating with the 
black hole to $\theta_{\text {inc }}=180$ degrees for equatorial orbits counterrotating with respect to the black hole, passing through $\theta_{\text {inc }}=90$ degrees for polar orbits.

In order to fix the initial conditions of a geodesic, let us first parametrize it with the Carter time $\lambda$, which is related to the proper time by [46]

$$
\frac{d \tau}{d \lambda}=\Sigma
$$

This is a very useful choice because it makes the geodesic equation separable [46]:

$$
\begin{array}{ll}
\left(\frac{d r}{d \lambda}\right)^{2}=V_{r}(r), & \frac{d t}{d \lambda}=V_{t}(r, \theta), \\
\left(\frac{d \theta}{d \lambda}\right)^{2}=V_{\theta}(\theta), & \frac{d \phi}{d \lambda}=V_{\phi}(r, \theta),
\end{array}
$$

with

$$
\begin{gathered}
V_{t}(r, \theta) / M^{2}=\tilde{E}\left[\frac{\left(\tilde{r}^{2}+\tilde{a}^{2}\right)^{2}}{\tilde{\Delta}}-\tilde{a}^{2} \sin ^{2} \theta\right] \\
+\tilde{a} \tilde{L}_{z}\left(1-\frac{\tilde{r}^{2}+\tilde{a}^{2}}{\tilde{\Delta}}\right) \\
V_{r}(r) / M^{4}=\left[\tilde{E}\left(\tilde{r}^{2}+\tilde{a}^{2}\right)-\tilde{a} \tilde{L}_{z}\right]^{2} \\
\quad-\tilde{\Delta}\left[\tilde{r}^{2}+\left(\tilde{L}_{z}-\tilde{a} \tilde{E}\right)^{2}+\tilde{Q}\right] \\
V_{\theta}(\theta) / M^{2}=\tilde{Q}-\tilde{L}_{z}^{2} \cot ^{2} \theta-\tilde{a}^{2}\left(1-\tilde{E}^{2}\right) \cos ^{2} \theta \\
V_{\phi}(r, \theta) / M=\tilde{L}_{z} \csc ^{2} \theta+\tilde{a} \tilde{E}\left(\frac{\tilde{r}^{2}+\tilde{a}^{2}}{\tilde{\Delta}}-1\right)-\frac{\tilde{a}^{2} \tilde{L}_{z}}{\tilde{\Delta}} .
\end{gathered}
$$

This means, in particular, that the $r$ and $\theta$ motions are periodic in $\lambda$. The initial conditions of the geodesic can then be characterized by the values $t_{0}$ and $\phi_{0}$ of the coordinates $t$ and $\phi$ when $\lambda=0$, the value $\lambda_{r 0}$ of the Carter time nearest to $\lambda=0$ at which $r\left(\lambda_{r 0}\right)=r_{\mathrm{p}}$, and the value $\lambda_{\theta 0}$ of the Carter time nearest to $\lambda=0$ at which $\theta\left(\lambda_{\theta 0}\right)=\theta_{\min }$ [42].

Let us first fix the geodesic under consideration by choosing the parameters $p, e$, and $\theta_{\text {inc }}$ so as to obtain a bound and stable orbit (see Ref. [49] for details) and by choosing the initial conditions as described above. One could in principle use Eqs. (43), (44), and (47) [or (50)] to compute the rates of change of $\tilde{E}, \tilde{L}_{z}$, and $\tilde{Q}$ due to the hydrodynamic drag as a function of the Carter time $\lambda$. However, because the time scale of the orbital evolution due to the interaction with the torus is much longer than the orbital period, we can apply the adiabatic approximation and compute instead the averages of $\dot{\tilde{E}}, \dot{\tilde{L}}_{z}$, and $\dot{\tilde{Q}}$ over times much longer than the orbital periods. This approximation is routinely adopted when studying the effect of radiation reaction on EMRIs [42-45], and it is easy to implement when one considers instead the effect of the hydrodynamic drag. Denoting, respectively, with \langle\rangle$_{t}$ and \langle\rangle$_{\lambda}$ the average over an infinite coordinate time and the average over an infinite Carter time, we can write [42]

$$
\langle\dot{\Psi}\rangle_{t}=\frac{\langle d \Psi / d \lambda\rangle_{\lambda}}{\langle d t / d \lambda\rangle_{\lambda}}
$$

where $\Psi$ is a placeholder for either $\tilde{E}, \tilde{L}_{z}$, or $\tilde{Q}$.

Using now Eq. (4) (with the assumption that the torus is symmetric with respect to the equatorial plane) in Eqs. (43), (44), and (47) [or (50)], it is easy to show that $d \tilde{E} / d \lambda, d \tilde{L}_{z} / d \lambda$, and $d \tilde{Q} / d \lambda$ depend, once fixed $\tilde{E}, \tilde{L}_{z}$, and $\tilde{Q}$, only on the $r$ and $\cos ^{2} \theta$ of the geodesic under consideration-i.e., $r=r\left(\lambda, \lambda_{r 0}\right)$ and $\cos ^{2} \theta=\cos ^{2} \theta\left(\lambda, \lambda_{\theta 0}\right)-$ and on the sign of $u_{r}^{\text {sat }}$, which we will denote by $\epsilon_{r}$. [The dependence on this sign arises because of the terms due to the radial drag, as the quantity $u_{r}^{\text {sat }}$ appearing in the definitions of $\boldsymbol{\sigma}$ and $\boldsymbol{\chi}$ can be expressed in terms of $r$ and $\cos ^{2} \theta$ using Eqs. (53) -(58) only up to such a sign.] Similarly, $d t / d \lambda$ is given by the geodesic equation (54) and depends, once fixed $\tilde{E}, \tilde{L}_{z}$, and $\tilde{Q}$, only on the $r$ and $\cos ^{2} \theta$ of the geodesic [cf. Eq. (55)].

Using now the fact that the $r$ and $\theta$ motions are periodic when expressed in the Carter time, we can expand the functions $d \tilde{E} / d \lambda, d \tilde{L}_{z} / d \lambda$ and $d \tilde{Q} / d \lambda$, and $d t / d \lambda$ appearing in Eq. (59) in a Fourier series. Noting that the oscillating terms average out, one can then write these equations using only averages of these functions over the $r$ and $\theta$ periods. More precisely, writing the $r$ and $\theta$ motions as $r\left(\lambda, \lambda_{r 0}\right)=\hat{r}\left(\lambda-\lambda_{r 0}\right)$ and $\theta\left(\lambda, \lambda_{\theta 0}\right)=\hat{\theta}\left(\lambda-\lambda_{\theta 0}\right)$ (where we have denoted with a "hat" a fiducial geodesic having the same $\tilde{E}, \tilde{L}_{z}$, and $\tilde{Q}$ as the geodesic under consideration and $\lambda_{r 0}=\lambda_{\theta 0}=0$ ), using the fact that $d \tilde{E} / d \lambda, d \tilde{L}_{z} / d \lambda$, and $d \tilde{Q} / d \lambda$ depend on $r, \cos ^{2} \theta$, and $\epsilon_{r}$, and using the fact that $d t / d \lambda$ depends only on $r$ and $\cos ^{2} \theta$, we can easily write Eq. (59) as [42]

$$
\langle\dot{\Psi}\rangle_{t}=\frac{\int_{0}^{\Lambda_{r}} d \lambda_{r} \int_{0}^{\Lambda_{\theta} / 4} d \lambda_{\theta} d \Psi / d \lambda\left(\hat{r}\left(\lambda_{r}\right), \cos ^{2} \hat{\theta}\left(\lambda_{\theta}\right), \epsilon_{r}\right)}{\int_{0}^{\Lambda_{r}} d \lambda_{r} \int_{0}^{\Lambda_{\theta} / 4} d \lambda_{\theta} V_{t}\left(\hat{r}\left(\lambda_{r}\right), \cos ^{2} \hat{\theta}\left(\lambda_{\theta}\right)\right)},
$$

where $\Psi$ is again a placeholder for either $\tilde{E}, \tilde{L}_{z}$, or $\tilde{Q}$. Note that here $\Lambda_{r}$ and $\Lambda_{\theta}$ are the $r$ and $\theta$ periods and that $d \tilde{E} / d \lambda, d \tilde{L}_{z} / d \lambda$ and $d \tilde{Q} / d \lambda$, and $d t / d \lambda$ are expressed using Eqs. (43), (44), (47) [or (50)], and (54)-(58). Using now the definitions of $\boldsymbol{\sigma}$ and $\boldsymbol{\chi}$ [Eqs. (31) and (32)], it is easy to check that the changes of $\tilde{E}, \tilde{L}_{z}$, and $\tilde{Q}$ arising from the radial drag average out in the above equation because of the presence of the sign $\epsilon_{r}$ (in particular $\left.\chi_{t}, \chi_{\phi}, \chi_{\theta} \propto \epsilon_{r}\right)$. As a result, one can assume $\dot{p}_{\text {defl }}^{\text {rad }}=0$ $a b$ initio when computing Eq. (60) and benefit from another small simplification since, as we have already mentioned, $d \tilde{E} / d \lambda, d \tilde{L}_{z} / d \lambda$, and $d \tilde{Q} / d \lambda$ would depend only on $r$ and $\cos ^{2} \theta$ if it were not for the radial drag, which 
brings in the dependence on $\epsilon_{r}$. With this assumption, all of the integrals appearing in (60) can therefore be performed over $\lambda_{r} \in\left[0, \Lambda_{r} / 2\right]$ rather than over $\lambda_{r} \in\left[0, \Lambda_{r}\right]$. Finally, note also that the rates of change (60) do not depend on the initial conditions $\lambda_{r 0}$ and $\lambda_{\theta 0}$ of the geodesic.

In order to reduce Eq. (60) to a form suitable for numerical integration, we can express our fiducial geodesic with the phase variables $\psi$ and $\chi$, defined by $[45,49,50]$

$$
\begin{aligned}
& \hat{r}(\psi)=\frac{p}{1+e \cos \psi}, \\
& \cos \hat{\theta}(\chi)=z_{-} \cos \chi .
\end{aligned}
$$

Note that $\psi$ and $\chi$ change by $2 \pi$ during, respectively, an $r$ and a $\theta$ period. Inserting then these definitions into the geodesic equation (54) one gets $[45,49,50]$

$$
\begin{gathered}
\frac{d \psi}{d \lambda}=\frac{p}{1-e^{2}} \sqrt{J(\psi),} \\
\frac{d \chi}{d \lambda}=M \sqrt{\beta\left(z_{+}-z_{-} \cos ^{2} \chi\right)},
\end{gathered}
$$

where

$$
\begin{gathered}
J(\psi)=\left(1-\tilde{E}^{2}\right)\left(1-e^{2}\right)+2\left(1-\tilde{E}^{2}-\frac{1-e^{2}}{\tilde{p}}\right) \\
\times(1+e \cos \psi)+(1+e \cos \psi)^{2}\left[\left(1-\tilde{E}^{2}\right) \frac{3+e^{2}}{1-e^{2}}\right. \\
\left.-\frac{4}{\tilde{p}}+\frac{1-e^{2}}{\tilde{p}^{2}}\left(\beta+\tilde{L}_{z}^{2}+\tilde{Q}\right)\right] \\
\beta=\tilde{a}^{2}\left(1-\tilde{E}^{2}\right), \\
z_{+}=\frac{\tilde{Q}+\tilde{L}_{z}^{2}+\beta+\sqrt{\left(\tilde{Q}+\tilde{L}_{z}^{2}+\beta\right)^{2}-4 \beta \tilde{Q}}}{2 \beta}
\end{gathered}
$$

with $\tilde{p}=p / M$. Note that $d \psi / d \lambda$ and $d \chi / d \lambda$, differently from $d r / d \lambda$ and $d \theta / d \lambda$, are nonzero at the inversion points of the $r$ and $\theta$ motions, making $\psi$ and $\chi$ very useful for numerical integration.

Changing the integration variables $\lambda_{r}$ and $\lambda_{\theta}$ to $\psi$ and $\chi$, Eq. (60) becomes

$$
\begin{aligned}
\langle\dot{\Psi}\rangle t= & \int_{0}^{\pi} d \psi \int_{0}^{\pi / 2} d \chi \\
& \times \frac{d \Psi /\left.d \lambda\right|_{\dot{p}_{\text {defl }}^{\text {rad }}=0}\left(\hat{r}(\psi), \cos ^{2} \hat{\theta}(\chi)\right)\left(1-e^{2}\right)}{p \sqrt{J(\psi) \beta\left(z_{+}-z_{-} \cos ^{2} \chi\right)}} \\
& \times\left[\int_{0}^{\pi} d \psi \int_{0}^{\pi / 2} d \chi \frac{V_{t}\left(\hat{r}(\psi), \cos ^{2} \hat{\theta}(\chi)\right)\left(1-e^{2}\right)}{p \sqrt{J(\psi) \beta\left(z_{+}-z_{-} \cos ^{2} \chi\right)}}\right]^{-1}
\end{aligned}
$$

Note that the two-dimensional integrals involved in these expressions can be easily computed numerically (e.g., iterating Romberg's method [51]) once fixed the orbital parameters $p, e, \theta_{\text {inc }}$ of the geodesic under consideration.

\section{RESULTS}

In this section we will consider constant $\ell$ tori around Kerr SMBHs and compare their influence on EMRIs with that of gravitational wave emission (i.e., radiation reaction) in the adiabatic approximation. In particular, we will compute the rates of change (68) of the energy, angular momentum and Carter constant due to the hydrodynamic drag, for circular equatorial orbits (Sec. IVA) and for generic (inclined and eccentric) orbits (Sec. IV B). Since $\tilde{E}, \tilde{L}_{z}$, and $\tilde{Q}$ can be expressed analytically as functions of the orbital parameters $p, e$, and $\theta_{\text {inc }}$ [49], it is then easy to compute the rates of change $d p / d t, d e / d t$, and $d \theta_{\text {inc }} / d t$ due to the hydrodynamic drag. For the same orbits, we will consider also the radiation reaction, for which we will use the kludge fluxes $d \tilde{E} / d t, d \tilde{L}_{z} / d t$, and $d \tilde{Q} / d t$ of Ref. [52] to compute $d p / d t$, $d e / d t$, and $d \theta_{\text {inc }} / d t$. Note that these kludge fluxes are a good approximation to the fluxes computed rigorously in the adiabatic approximation with the Teukolsky-Sasaki-Nakamura formalism [45,53]. In fact, since they are based on a post-Newtonian expansion corrected with fits to fluxes computed with the TeukolskySasaki-Nakamura formalism for circular orbits, these kludge fluxes are accurate within 3\% for circular orbits and their accuracy is expected to be within $10-15 \%$ also for generic orbits with $p \gtrsim 6 M$. Moreover, they are expected to be off at most by $25-30 \%$ even for smaller values of the semilatus rectum $p$ (cf. Ref. [52], Table I).

The mass of the $\mathrm{SMBH}$ is fixed to $M=10^{6} M_{\odot}$ while its spin parameter $a$ ranges from $-0.998 M$ to $0.998 M$ (note that $|a|=0.998 M$ is a reasonable upper limit for the spin attainable as the result of mass accretion [54] or binary black hole mergers $[55,56])$, and the mass of the satellite black hole is instead $m=1 M_{\odot}$. The constant $\ell$ torus is assumed to be composed of an isentropic monatomic gas (i.e., a $\Gamma=5 / 3$ polytrope) and is considered to be exactly filling its outermost closed equipotential surface $(\Delta W=$ 0 ), so as to present a zero accretion rate $\dot{M}$ onto the SMBH. ${ }^{8}$ Once assumed $\Delta W=0$ and $\Gamma=5 / 3$, the specific angular momentum of the torus is uniquely fixed by choosing the outer radius. A reasonable outer radius for a realistic accretion disk is given by $r_{\text {out }}=10^{5} \mathrm{M}$ [14], and this is indeed the value that we will use for most of our analysis, although we will briefly consider also different values for

\footnotetext{
${ }^{8}$ While realistic thick disks are generally expected to accrete onto the SMBH, these configurations are clearly nonstationary and cannot therefore be reproduced within our framework. However, it is easy to show that if one cuts off a torus solution with $\dot{M}>0$ at $r=r_{\text {cusp }}$, the effect of the satellite-torus interaction will be enhanced with respect to the $\dot{M}=0$ solution having the same mass and outer radius: the choice $\dot{M}=0$ is thus useful to obtain at least a lower limit for the effect of the satellite-torus interaction on EMRIs.
} 
$r_{\text {out }}$ in order to study the impact of this parameter on the final results. The polytropic constant $\kappa$ of the equation of state is finally fixed by the requirement that $M_{\mathrm{t}}=0.1 M$. While this could be a reasonable value for the mass of a realistic accretion disk in AGNs [14], we will see that our results scale proportionally to $M_{\mathrm{t}}$, thus allowing one to extrapolate them easily to the case $M_{\mathrm{t}}=M$, which is certainly an astrophysically plausible value, but one for which our test-fluid approach is no longer valid.

We should stress, however, that our results, when expressed in terms of the dimensionless orbital parameters $p / M, e$, and $\theta_{\text {inc }}$ are approximately independent of the mass $M$ of the SMBH and of the mass $m$ of the satellite black hole (provided that $M_{\mathrm{t}} / M$ and $r_{\text {out }} / M$ are maintained constant). Indeed, since the ratios between the rates of change $d p / d t, d e / d t$, and $d \theta_{\text {inc }} / d t$ due to the hydrodynamic drag and radiation reaction are of course dimensionless, it is not restrictive to fix $M=1$, because this simply corresponds to choosing a system of units. Note, in particular, that this means that systems with different $m$ and $M$ but equal mass ratio $m / M$ give exactly the same ratios between the rates of change $d p / d t, d e / d t$, and $d \theta_{\text {inc }} / d t$ due to the hydrodynamic drag and radiation reaction. Moreover, these rates of change are proportional to $m$ (in the case of the hydrodynamic drag this can be seen from Eqs. (27), (33), (34), (43), (44), and (47), while in the case of radiation reaction see for instance Ref. [52]), so this dependence on $m$ cancels out when taking the ratio. The only dependence on $m$ arises from the cutoff $r_{\min } \sim$ $2 m\left(1+v^{2}\right) / v^{2}$ appearing in Eq. (35), but this dependence clearly comes about only for supersonic velocities and is a logarithmic one. As such, the results which we present in this paper, although derived in the case of $m=1 M_{\odot}$ and $M=10^{6} M_{\odot}$, are also valid for $m=0.1 M_{\odot}$ and $M=$ $10^{5} M_{\odot}$ (exactly) or for $m=1 M_{\odot}$ and $M=10^{5} M_{\odot}$ (ex- actly for subsonic motion, and approximately - with an error comparable with those affecting the fit (35) or the kludge fluxes - for supersonic motion).

In all of our analysis we will focus on the region close to the SMBH $(r \lesssim 50 M)$, which contains only a small fraction of the mass of the torus (e.g., in the case of the model A1 of Table I, the mass contained in a radius $r=50 \mathrm{M}$ amounts to about $2.9 \times 10^{-5} M_{\mathrm{t}}$, and this fraction scales approximately as $r_{\text {out }}^{-3 / 2}$ when considering tori with different outer radii). This is the region relevant for gravitational-wave experiments like LISA. In particular, an EMRI's signal is expected to be detectable by LISA when its frequency (which is twice the orbital frequency) increases above $\approx 2 \mathrm{mHz}$ (below this frequency, in fact, there is a strong unresolvable foreground noise due to double white-dwarf binaries in our Galaxy [57]). This translates into a distance from the SMBH of $r \approx 10 M$ for $M=10^{6} M_{\odot}$, and to $r \approx 45 M$ for $M=10^{5} M_{\odot}$.

It should be noted, however, that the amplitude of an EMRI's signal scales with the distance from the SMBH: for a circular orbit of radius $r$, the Keplerian frequency is $2 \pi \nu=M^{1 / 2} /\left(r^{3 / 2} \pm a M^{1 / 2}\right) \approx M^{1 / 2} / r^{3 / 2}$ and the amplitude of the signal is $h \sim(m / D)(2 \pi \nu M)^{2 / 3} \sim(m / D)(M / r)$ [58], where $D$ is the distance from the observer to the source. As such, an EMRI around a $10^{5} M_{\odot} \mathrm{SMBH}$ will have a gravitational-wave amplitude that at $r \sim 45 M$ is about 10 times smaller than at $r \sim 5 M$. Therefore, to see the details of the waveforms at $r \sim 45 M$ the source must be $\sim 10$ times closer to us, which translates into a detection volume decreased by a factor $\sim 1000$. Nevertheless, this decrease of the detection volume may be compensated (at least partly) by the fact that the event-rate estimates consider only EMRIs in the strong-field region of the SMBH, even when $M=10^{5} M_{\odot}$ [3]. As such, since EMRIs in the early part of the inspiral are more numerous than those in

TABLE I. Models analyzed in this paper: all of them have $M=10^{6} M_{\odot}, \Gamma=5 / 3$ and are filling exactly their outermost closed equipotential surface (i.e., they have $\Delta W=0$ ). All the parameters are defined in Sec. II, except the average rest-mass density $\rho_{0 \text { avg }}=$ $M_{\mathrm{t} 0} / V$, where $V=\int_{\rho_{0}>0} \sqrt{-g} d^{3} x$. Note that the specific angular momentum of the torus needs to be tuned with high accuracy in order to obtain large outer radii such as those considered in these models, and for this reason we report $\ell / M$ with 10 decimal digits.

\begin{tabular}{|c|c|c|c|c|c|c|c|c|c|}
\hline lodel & $a$ & $M_{\mathrm{t}} / M$ & $r_{\text {out }} / M$ & $\kappa(\mathrm{CGS})$ & $\ell / M$ & in $/ M$ & $r_{\text {center }} / M$ & $\rho_{\text {center }}\left(\mathrm{g} / \mathrm{cm}^{3}\right)$ & $\rho_{\text {0avg }}\left(\mathrm{g} / \mathrm{cm}^{3}\right)$ \\
\hline$x$ & 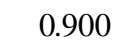 & 00 & $00 \times 10$ & $\times 10$ & 63245 & 73246 & 3.60963 & $.060 \times 10^{-5}$ & $1.475 \times 10^{-11}$ \\
\hline 2 & -0.900 & 100 & $1.000 \times 10^{5}$ & $4.198 \times 10^{22}$ & $756731^{\prime}$ & 5.65700 & 15.58890 & $2 \times 10^{-6}$ & $1.476 \times 10^{-11}$ \\
\hline 1 & 0.998 & 00 & $0 \times 1$ & $4.200 \times 10^{22}$ & .0894422310 & 1.09144 & 1.56484 & & \\
\hline 2 & 0 & 0 & & & & 425 & 58 & & $10^{-11}$ \\
\hline 3 & 00 & 00 & $1.000 \times 1$ & $4.192 \times 10^{22}$ & 9999 & 4.00008 & 174 & & $0^{-11}$ \\
\hline 4 & -0.500 & 00 & & & & 62 & 47 & & )$^{-11}$ \\
\hline r & -0.998 & 100 & $1.000 \times 10^{5}$ & $4.188 \times 10^{22}$ & $4.826 \mathrm{C}$ & 21 & 218 & $96 \times 10^{-6}$ & $10^{-11}$ \\
\hline 1 & 0.900 & 00 & $\times 10^{3}$ & $\times 10^{20}$ & 2.6 & 18 & 22 & & $0^{-5}$ \\
\hline 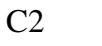 & -0.900 & 100 & $1.000 \times 10^{3}$ & $3.607 \times 10^{20}$ & 4.749056 & 5.67540 & 760 & $75 \times 10^{-3}$ & $0^{-5}$ \\
\hline & & 00 & 1.00 & $\times 10^{21}$ & 2.6324 & 253 & 32 & -3 & $0^{-8}$ \\
\hline 4 & -0.900 & 0.100 & $1.000 \times 10^{4}$ & $4.103 \times 10^{21}$ & 4.7560304461 & 5.65866 & 15.58057 & $1.297 \times 10^{-4}$ & $1.489 \times 10^{-8}$ \\
\hline 5 & 0.900 & 0.100 & & $4.201 \times 10^{23}$ & 2.6324549842 & 1.73246 & 3.60967 & $1.283 \times 10^{-6}$ & $1.475 \times 10^{-14}$ \\
\hline 6 & -0.900 & 0.100 & $1.000 \times 10^{6}$ & $4.199 \times 10^{23}$ & 4.7568019526 & 5.65683 & 15.58974 & $1.258 \times 10^{-7}$ & $1.475 \times 10^{-14}$ \\
\hline
\end{tabular}


the strong-field region, one expects to see a number of these events larger than the naive estimate given by the rate expected for strong-field EMRIs around a $10^{5} M_{\odot} \mathrm{SMBH}$ divided by the detection volume decrease factor $\sim 1000$. Of course, the event rates could be even larger if the satellite were a black hole with $m \sim 100 M_{\odot}$, because the amplitude of the signal is proportional to $m$, but too little is presently known about these objects to draw any sound conclusions (see for instance Ref. [59] for a review on intermediatemass black holes as possible sources for LISA).

\section{A. Circular equatorial orbits}

The evolution of circular equatorial orbits is very simple in the adiabatic approximation. As mentioned in Sec. III A, both the radiation reaction and the hydrodynamic drag maintain circular orbits circular and, due to the symmetry of the Kerr spacetime and of the torus with respect to the equatorial plane, equatorial orbits will remain equatorial. Therefore, the evolution of circular equatorial orbits under both radiation reaction and hydrodynamic drag can be characterized with only one quantity (the rate of change of the radius $d r / d t$ ), to which the rates of change of the energy and angular momentum, $d \tilde{E} / d t=(d \tilde{E} / d r)(d r / d t)$ and $d \tilde{L}_{z} / d t=\left(d \tilde{L}_{z} / d r\right)(d r / d t)$, are proportional. $(d \tilde{Q} / d t$ is instead identically zero for equatorial orbits.) Moreover, one does not need to compute the infinite-time averages (68), because the rates of change of $\tilde{E}, \tilde{L}_{z}$, and $\tilde{Q}$ due to the hydrodynamic drag, given by Eqs. (43), (44), and (47) [or (50)], are already functions of the orbital radius alone. (Note also that the 4-vector $\boldsymbol{\chi}$ reduces to $\boldsymbol{\chi}=-\partial_{r} / \sqrt{g_{r r}}$.)

The ratio between the rates of change of the orbital radius due to hydrodynamic drag and radiation reaction is a convenient measure of the "efficiency" of the hydrodynamic drag. Defining this quantity simply as $\beta_{\dot{r}} \equiv$ $(d r / d t)_{\text {hydro }} /(d r / d t)_{\mathrm{GW}}$, we show in the top plot of Fig. 1 the absolute value of $\beta_{\dot{r}}$ as a function of the radius $r$ of circular equatorial bound stable orbits. The two curves refer to models A1 and A2 of Table I, and are labeled with the spin parameter $a$ of the SMBH. Note that in order to present all the data in a single plot, a positive $r$ refers to orbits rotating in the positive $\phi$ direction ("prograde orbits" i.e., corotating with the torus), while a negative $r$ refers to orbits rotating in the negative $\phi$ direction ("retrograde orbits" i.e., counterrotating with respect to the torus). The middle plot compares the velocity $v$ of the satellite in the rest frame of the fluid with the sound velocity $v_{s}$, while the bottom plot shows instead the energy density $\rho$ of the torus. Note that in all the plots the vertical axis is drawn in logarithmic scale. As such, the vertical asymptotes appearing in Fig. 1 (as well as in Figs. 2 and 3, which are in logarithmic scale too) actually correspond to a zero value for the quantity under consideration.

Note that if the torus is corotating with the black hole, the radius of the innermost stable circular orbit (ISCO) is always larger than the inner radius of the torus, both for prograde and retrograde orbits. For prograde orbits, this immediately follows from the considerations of Sec. II (since our tori have $\Delta W=0$ and $\ell_{\mathrm{ms}}<\ell<\ell_{\mathrm{mb}}$, we have $r_{\text {in }}=r_{\text {cusp }}<r_{\mathrm{ms}}$, and $r_{\mathrm{ms}}$ is exactly the radius of the prograde ISCO), while for retrograde orbits it is sufficient to note that the retrograde ISCO is located at a larger radius than the prograde one. Bearing this in mind, it is then easy to understand why none of the quantities plotted in Fig. 1 for model A1 $(a=0.9 M)$ goes to zero when approaching the SMBH: although the density, the velocity of the satellite relative to the torus, the sound velocity, and $(d r / d t)_{\text {hydro }}$ are exactly zero at the inner radius of the torus, this radius is smaller than that of the ISCO and therefore no bound stable orbits exist there.

If instead the torus is counterrotating with respect to the black hole (i.e., $a<0$ ), the radius of the ISCO is larger than $r_{\text {in }}$ for prograde orbits (this follows again from $r_{\text {in }}=$ $r_{\text {cusp }}<r_{\text {ms }}$ ), but it is not possible to conclude that the radius of the ISCO is larger than $r_{\text {in }}$ also for retrograde orbits. In fact, the ISCO counterrotating with respect to the torus (i.e., the "retrograde" ISCO) is corotating with the black hole and thus lies at a radius smaller than the "prograde" ISCO. Indeed, for the model A2 $(a=-0.9 M)$ considered in Fig. 1 the retrograde ISCO is at a radius smaller than $r_{\text {in }}$. As a consequence, the density, the sound velocity, and $(d r / d t)_{\text {hydro }}$ for model A2 go to zero when the radius of the retrograde orbits decreases, being in fact zero at the inner edge of the torus. (Of course, the velocity of the satellite relative to the fluid does not go to zero when the radius of the retrograde orbits decreases, because the satellite and the torus are rotating in opposite directions.)

As it can be seen in the top plot, the ratio $\left|\beta_{\dot{r}}\right|$ is larger for the retrograde orbits than for the prograde ones. The reason can be easily understood from the middle plot, which shows that the retrograde motion is always supersonic. As such, the long-range drag, which increases significantly when passing from the subsonic to the supersonic regime [cf. Eq. (35)], enhances the torussatellite interaction for the retrograde orbits. From the middle plot one can also note that relativistic velocities $(v \gtrsim 0.6)$ are reached in the case of retrograde orbits very close to the SMBH, thus further enhancing the hydrodynamic drag because of the relativistic correction factor $\gamma^{2}\left(1+v^{2}\right)^{2}$ appearing in Eq. (33). However, we should note that when $v$ reaches its maximum value (i.e., $v \sim 0.8$ for model A2) the effect of the relativistic correction factor on the hydrodynamic drag is hindered by the small value of the density, which goes to zero at the inner edge of the torus (cf. the bottom plot).

As it can be easily understood from the formulas reviewed in Sec II, a change in the polytropic constant $\kappa$ leaves all the parameters of the torus unchanged, except the energy density $\rho$, the rest mass density $\rho_{0}$, the pressure $p$ [all of which scale proportionally to $\kappa^{-1 /(\Gamma-1)}$ ], and the total mass energy and rest mass, $M_{\mathrm{t}}$ and $M_{\mathrm{t} 0}$ (which scale 

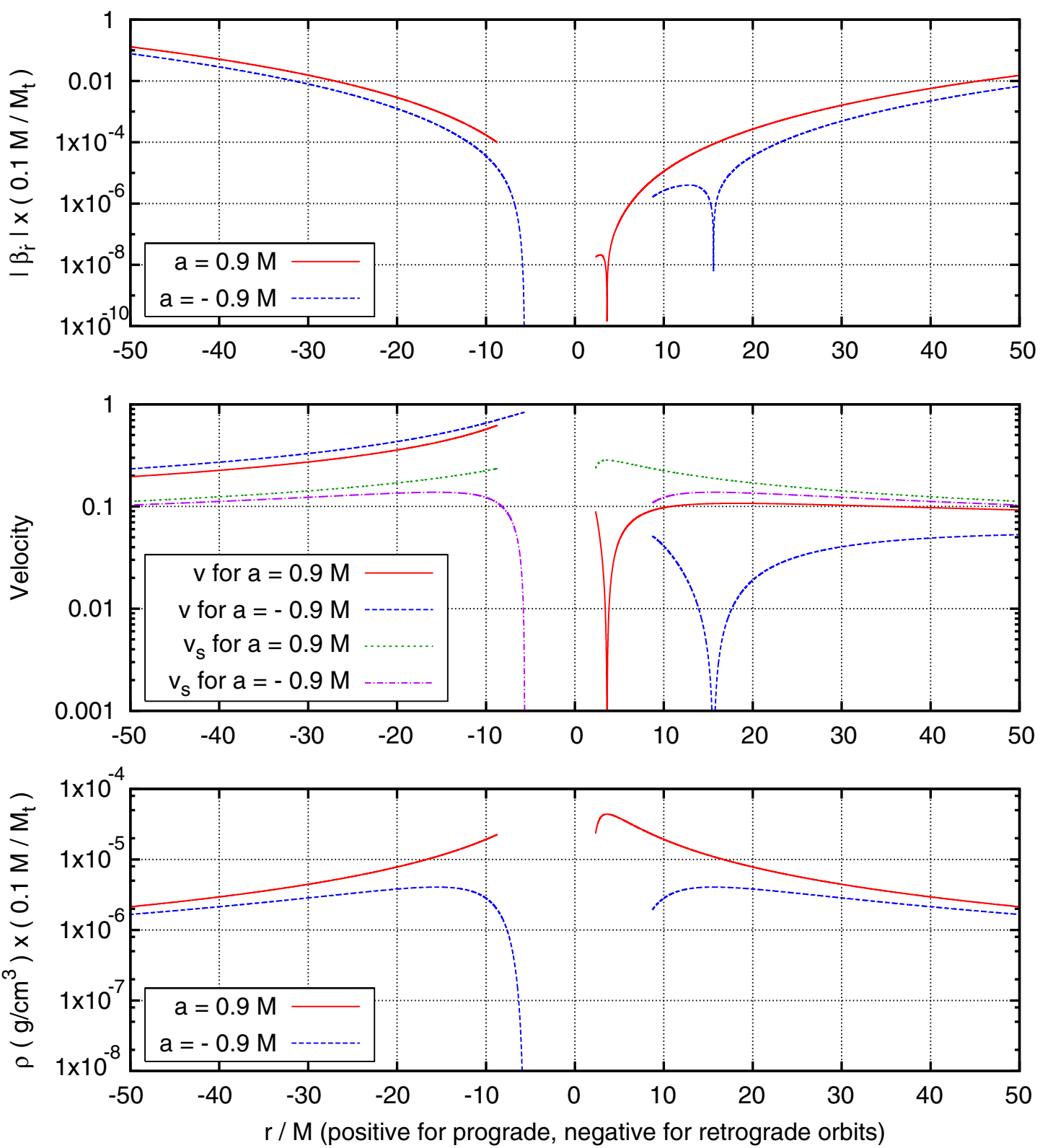

FIG. 1 (color online). The top plot shows the absolute value of the ratio $\beta_{\dot{r}} \equiv(d r / d t)_{\mathrm{hydro}} /(d r / d t)_{\mathrm{GW}}$ between the rates of change of the orbital radius due to hydrodynamic drag and radiation reaction, for circular bound stable orbits in the equatorial plane, as a function of the radius $r$. For graphical reasons $r$ is considered positive for orbits in the positive $\phi$ direction ("prograde orbits" i.e., corotating with the torus) and negative for those in the negative $\phi$ direction ("retrograde orbits" i.e., counterrotating with respect to the torus). Note that all the curves of this figure (including those of the middle and bottom plots) are terminated at the (prograde or retrograde) ISCO. The middle plot compares the velocity $v$ of the satellite in the rest frame of the fluid with the sound velocity $v_{s}$, while the bottom plot shows the energy density of the torus. The curves refer to the models A1 and A2 of Table I, which are labeled here with the spin parameter $a$ of the SMBH. Note that in all the plots the vertical axis is drawn in logarithmic scale. As such, the vertical asymptotes appearing in these plots actually correspond to a zero value for the quantity under consideration.

proportionally to $\rho$ ). For this reason, the bottom plot of Fig. 1 scales linearly with the mass of the torus (cf. the label of the vertical axis). Noting, from the formulas of Sec. III A, that the rates of change of the $\tilde{E}, \tilde{L}_{z}$, and $\tilde{Q}$ are proportional to the energy density $\rho$, the same scaling applies to the top plot. This is a very useful feature, because although the value used for the figures of this paper-i.e.,
$M_{\mathrm{t}}=0.1 M$ - could be a plausible mass for the torus, very little is known about these objects and larger or smaller masses may be possible. In general, a different mass $M_{\mathrm{t}}$ could have important effects. For instance, extrapolating to the case $M_{\mathrm{t}}=M$, in which our test-fluid approximation is no longer valid, the ratio $\left|\beta_{\dot{r}}\right|$ would be enhanced by a factor 10 , and for orbits counterrotating with respect to the 


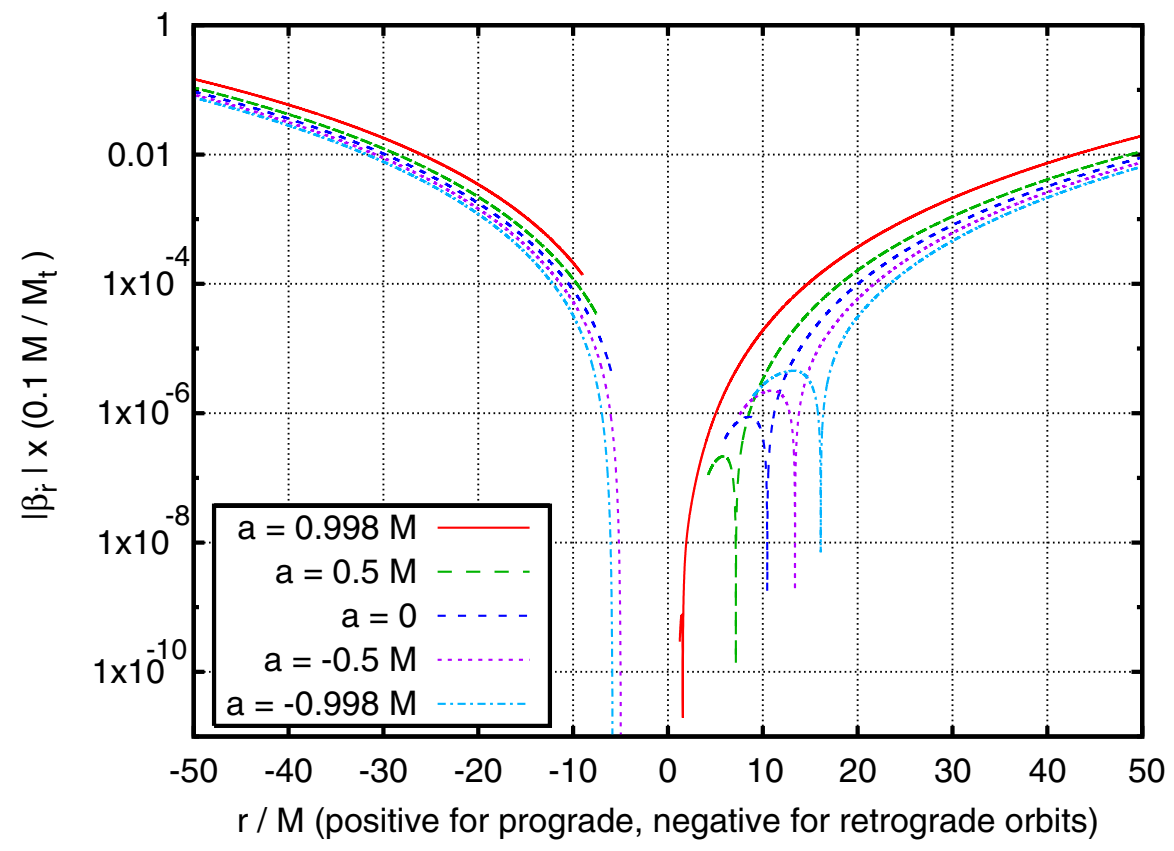

FIG. 2 (color online). The same as the top plot of Fig. 1, but for the models B1-B5 of Table I, which are labeled here with the spin parameter $a$ of the SMBH.

torus with $r \gtrsim 40 M$ the effects of hydrodynamic drag and radiation reaction would become comparable. ${ }^{9}$

It is also worth pointing out that at the center of the torus $(d r / d t)_{\text {hydro }}$ changes sign for prograde orbits, being negative for $r>r_{\text {center }}$ and positive for $r<r_{\text {center }}\left[(d r / d t)_{\mathrm{GW}}\right.$ is instead always negative because gravitational waves carry a positive amount of energy away from the source.] This change of sign corresponds, in the top plot of Fig. 1, to the zero value for $\left|\beta_{\dot{r}}\right|$. This behavior comes about because, although the density reaches its maximum at the center, the motion of the fluid is exactly Keplerian (geodesic) there, and the relative velocity of the satellite is therefore exactly zero (cf. the middle plot). This means, in particular, that $\tilde{E}=-u_{t}^{\text {sat }}=-u_{t}^{\text {fluid }}=U$, which together with Eq. (43) and $\chi_{t}=0$ gives $\dot{\tilde{E}}=0$ and therefore $\dot{r}=0$ for prograde orbits at $r=r_{\text {center }}$. Moreover, if $r>r_{\text {center }}$ the specific angular momentum of the satellite is larger than that of the torus (cf. for instance Ref. [33], Fig. 5), and therefore the satellite is slowed down by the interaction with the fluid (i.e., $\dot{r}<0$ ). On the other hand, if $r<r_{\text {center }}$ the specific angular momentum of the satellite is smaller than that of the torus, and the satellite is speeded up (i.e., $\dot{r}>0$ ).

Figure 2 shows the absolute value of $\beta_{\dot{r}}$ as a function of the radius $r$ of circular equatorial bound stable orbits for

\footnotetext{
${ }^{9}$ Note, however, that even values of $\left|\beta_{\dot{r}}\right|$ less than 1 can produce features detectable by LISA, because the dephasing time scales as $\sim\left|\dot{r}_{\mathrm{GW}}\right|^{-1 / 2}$ [60]. For instance, if $\left|\beta_{\dot{r}}\right| \approx 0.1$ the dephasing time between a waveform with only radiation reaction included and one with also the effect of the hydrodynamic drag is expected to be only $\sim 3$ times larger than the dephasing time between waveforms with and without radiation reaction included.
}

the models B1-B5 of Table I, whose spin parameter $a$ goes from $-0.998 M$ to $0.998 M$. As it can be seen, the situation is qualitatively very similar to the one presented in the top plot of Fig. 1. In particular, the effect of the hydrodynamic drag can be comparable to that of radiation reaction, but only if we extrapolate to $M_{\mathrm{t}}=M$ and, even in that case, only for orbits counterrotating with respect to the torus and with $r \gtrsim 40 M$.

We can also note that the effect of the spin $a$ on the results is negligible, except for the prograde orbits between the center and the ISCO, for which $\left|\beta_{\dot{r}}\right|$ decreases as $a$ increases. The reason for this can be easily understood by considering a satellite moving on a prograde circular equatorial orbit between the center and the inner edge of the torus, and by recalling that the difference between $\ell_{\mathrm{mb}}$ and $\ell_{\mathrm{ms}}$ represents an upper limit for the deviation of the specific angular momentum of the satellite away from that of the torus (see Ref. [33], Fig. 5). Because this deviation regulates the exchange of angular momentum between the torus and the satellite [cf. Eq. (44), where $\chi_{\phi}=0$ for circular orbits] and thus the rate of change of the orbital radius, $\beta_{\dot{r}}$ must go to zero if $\ell_{\mathrm{mb}}-\ell_{\mathrm{ms}}$ goes to zero. Since it is easy to verify that $\ell_{\mathrm{mb}}-\ell_{\mathrm{ms}} \rightarrow 0$ as $a \rightarrow$ $M$ [cf. Eq. (15)], it is natural to find that $\left|\beta_{\dot{r}}\right|$ decreases as $a$ increases.

Finally, in Fig. 3 we plot again $\left|\beta_{\dot{r}}\right|$, but for models A1A2 and C1-C6 of Table I, in which we have considered different values for the outer radius $r_{\text {out }}$ of the torus, ranging from $10^{3} M$ to $10^{6} M$. The reason for this is that although $r_{\text {out }} \sim 10^{5} M$ is a plausible value for the outer radius, little is known about the size of astrophysical accretion disks and larger or smaller outer radii may also 


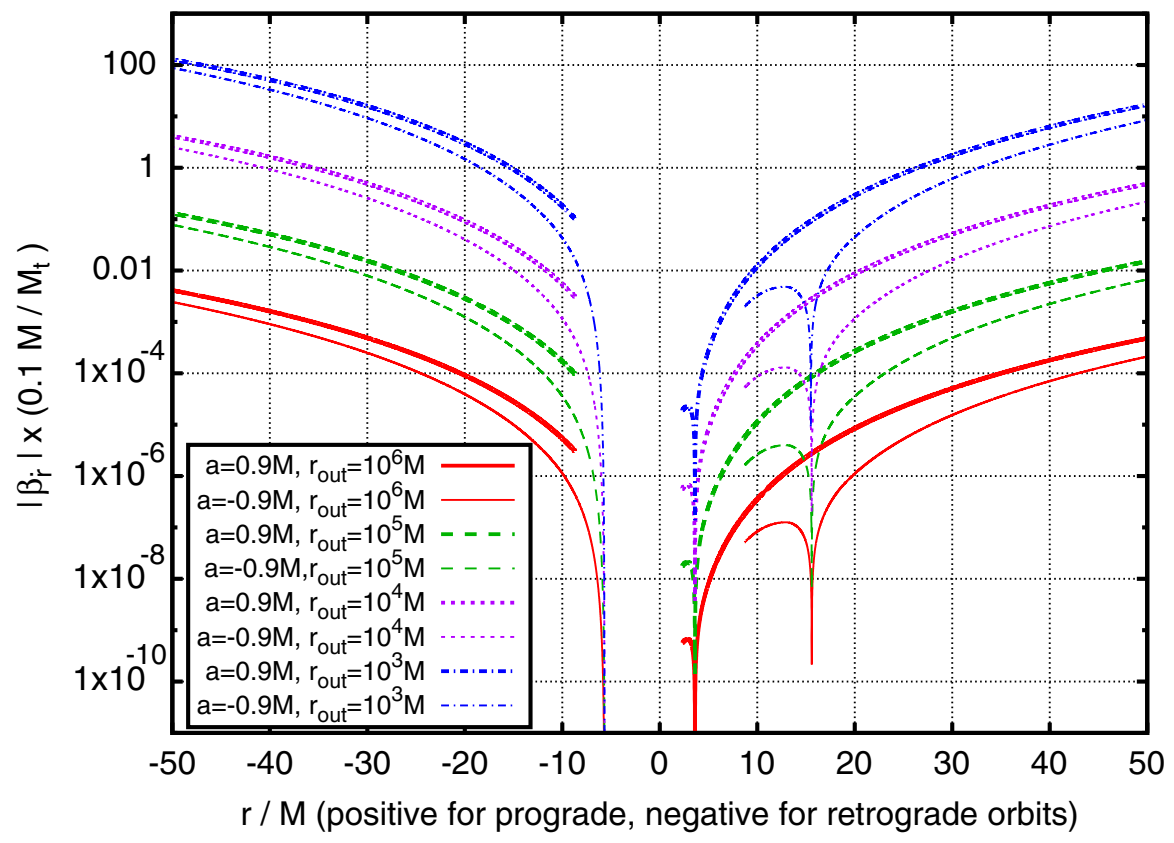

FIG. 3 (color online). The same as the top plot of Fig. 1, but for the models A1-A2 and C1-C6 of Table I, which are labeled here with the spin parameter $a$ of the SMBH and the outer radius $r_{\text {out }}$ of the torus.

be possible. As it can be seen from Fig. 3, a different outer radius will have significant effects for prograde orbits with $r \gtrsim 20 M$ and retrograde orbits with $r \gtrsim 10 M$, for which the effect of the hydrodynamic drag can become comparable to that of radiation reaction. In general, $\left|\beta_{\dot{r}}\right|$ progressively increases as the outer radius is decreased. This is rather simple to explain: decreasing $r_{\text {out }}$ while keeping $M_{\mathrm{t}}$ constant amounts to increasing the average rest-mass density and hence the hydrodynamic drag. When considered from this point of view, the uncertainty on the value of $r_{\text {out }}$ has an effect opposite to the uncertainty about the mass of the torus: a decrease of $r_{\text {out }}$ (or an increase of $M_{\mathrm{t}}$ ) induces an increase of $\left|\beta_{\dot{r}}\right|$. For circular orbits, this overall uncertainty can be easily modeled in terms of a simple scaling of the type

$$
\left.\beta_{\dot{r}}\left(r_{\text {out }}\right) \approx \beta_{\dot{r}}\right|_{5}\left(\frac{10^{5} M}{r_{\text {out }}}\right)^{3 / 2},
$$

where $\left.\beta_{\dot{r}}\right|_{5}$ is the efficiency for $r_{\text {out }}=10^{5} \mathrm{M}$. Note that the scaling power is not 3 as one may naively expect. This is because $\beta_{\dot{r}}$ is most sensitive to the changes of the rest-mass density in the inner part of the torus and this does not scale simply as $r_{\text {out }}^{-3}$.

\section{B. Generic orbits}

We will now extend the analysis of Sec. IVA to bound stable generic (inclined and eccentric) orbits. Although such an extension is in principle straightforward using the formulas introduced in Sec. III B, the space of parameters and results which one needs to examine greatly enlarges. Not only are generic orbits characterized by the three parameters $p, e, \theta_{\text {inc }}$ defined by Eq. (52), but one also needs to consider three quantities describing the evolution of each single orbit in the parameter space i.e., the rates of change $d p / d t, d e / d t$, and $d \theta_{\text {inc }} / d t$.

To simplify our analysis, we will focus mainly on model A1 of Table I, which could be a representative example of an astrophysical torus in an AGN, and then examine how the rates of change $d p / d t, d e / d t$, and $d \theta_{\text {inc }} / d t$ due to the hydrodynamic drag compare to those due to radiation reaction throughout the space of parameters $\left(p, e, \theta_{\text {inc }}\right)$. The considerations that we will draw for model A1 can, however, be extended simply to the cases of different masses and radii for the torus. As in the case of circular orbits, in fact, a larger (smaller) mass $M_{\mathrm{t}}$ for the torus when $r_{\text {out }}$ is held constant would simply increase (decrease) the rates $d p / d t, d e / d t$, and $d \theta_{\text {inc }} / d t$ due to the hydrodynamic drag by a factor $M_{\mathrm{t}} /(0.1 M)$. This scaling is exact (as long as the torus is not self-gravitating) and comes about because the rates of change of $\tilde{E}, \tilde{L}_{z}$, and $\tilde{Q}$ (and consequently those of $p, e$, and $\left.\theta_{\text {inc }}\right)$ are proportional to the energy density $\rho \propto M_{\mathrm{t}}$. Similarly, variations of $r_{\text {out }}$ will result in an effect which is similar to the one discussed for Fig. 3 in the case of circular orbits [cf. Eq. (69)], as we will see at the end of this section.

All of the results presented in this section have been computed by integrating numerically Eq. (68) using an iterated Romberg method [51], with a typical accuracy, depending on the parameters of the orbit under consideration, of $10^{-7}-10^{-4}$ and never worse than $4 \times 10^{-3}$. $^{10}$

\footnotetext{
${ }^{10}$ Note that the accuracy of the numerical integration is certainly adequate, because it is considerably better than the errors affecting the fit (35) as well as those affecting the kludge fluxes that we use to study the effects of radiation reaction.
} 


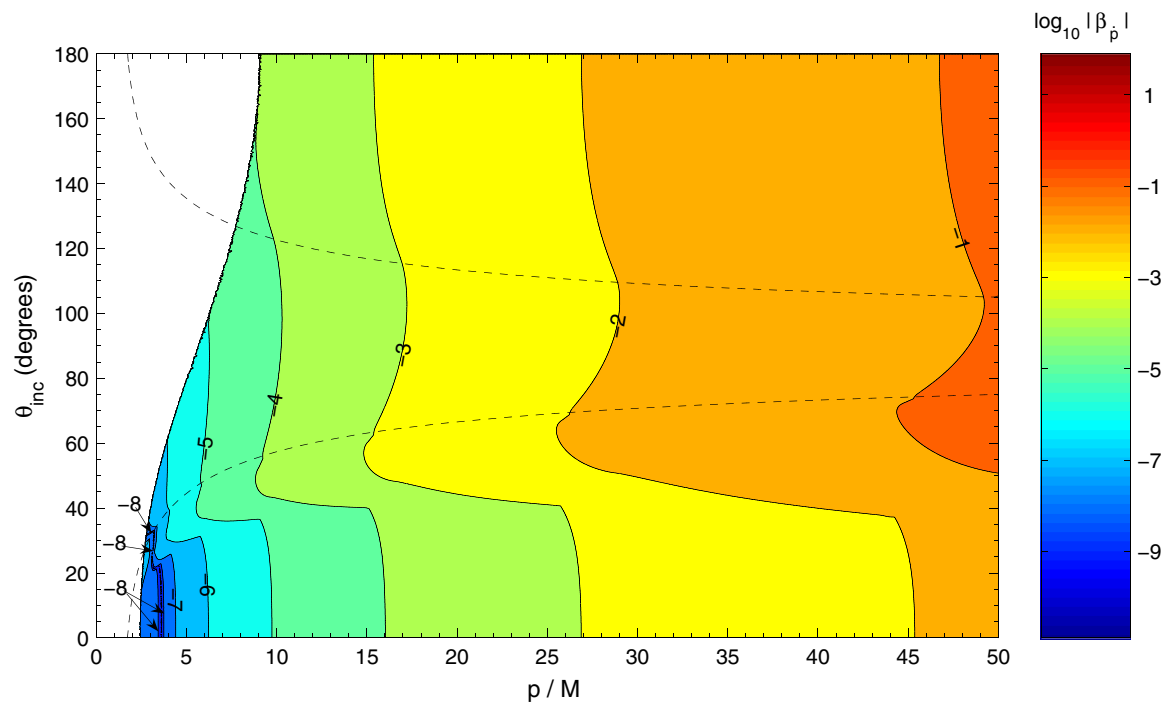

FIG. 4 (color online). $\quad \log _{10}\left|\beta_{\dot{p}}\right|$ for inclined orbits with $e=0.1$ as a function of the semilatus rectum $p$ and of the inclination angle $\theta_{\text {inc }}$. The figure refers to the model A1 of Table I, but a larger (smaller) mass $M_{\mathrm{t}}$ for the torus would simply increase (decrease) the absolute ratio $\left|\beta_{\dot{p}}\right|$ by a factor $M_{\mathrm{t}} /(0.1 M)$. The dashed line marks the edge of the torus.

We start by analyzing in detail orbits with eccentricity $e=0.1$, and we will then study the effect of a different eccentricity on the results. In Figs. 4-6 the color code and the contour levels show the base-10 logarithm of the "efficiencies" of $\dot{p}, \dot{e}$, and $\dot{\theta}_{\text {inc }}$, i.e., $\beta_{\dot{p}} \equiv \mid(d p / d t)_{\text {hydro }} /$ $(d p / d t)_{\mathrm{GW}}\left|, \beta_{\dot{e}} \equiv\right|(d e / d t)_{\mathrm{hydro}} /(d e / d t)_{\mathrm{GW}} \mid$, and $\beta_{\dot{\theta}_{\text {inc }}} \equiv$ $\left|\left(d \theta_{\text {inc }} / d t\right)_{\mathrm{hydro}} /\left(d \theta_{\mathrm{inc}} / d t\right)_{\mathrm{GW}}\right|$, as functions of $p$ and $\theta_{\mathrm{inc}}$. The dashed line marks the "edge of the torus" i.e., the location in the $\left(p, \theta_{\text {inc }}\right)$ plane of the orbits having $\theta_{\text {inc }}=$ $\theta_{\text {inc, } t}(p)$, where $\theta_{\text {inc, } t}(r)$ is the function giving the angle between the surface of the torus and the equatorial plane in terms of the radius $r$. The blank part on the left portion of these figures refers to the region where no bound stable orbits exist, and we will refer to the line marking the boundary of this region as the separatrix [61]
Each figure has been obtained by computing the quantity under consideration using Eq. (68) for $\sim 5 \times 10^{5}$ orbits irregularly distributed in the $\left(p, \theta_{\text {inc }}\right)$ plane, and then linearly interpolating on a grid of $1500 \times 1500$ nodes using a Delaunay triangle-based method. The gridded data obtained in this way have been used to draw the contour levels. Not surprisingly, Figs. 4-6 show somewhat the same trend as the results presented in Sec. IVA for circular equatorial orbits, with the effect of the torus becoming comparable to that of the radiation reaction far away from the black hole and becoming instead negligible in the strong-field region of the black hole. However, these figures present also a variety of features that we will now analyze in detail.

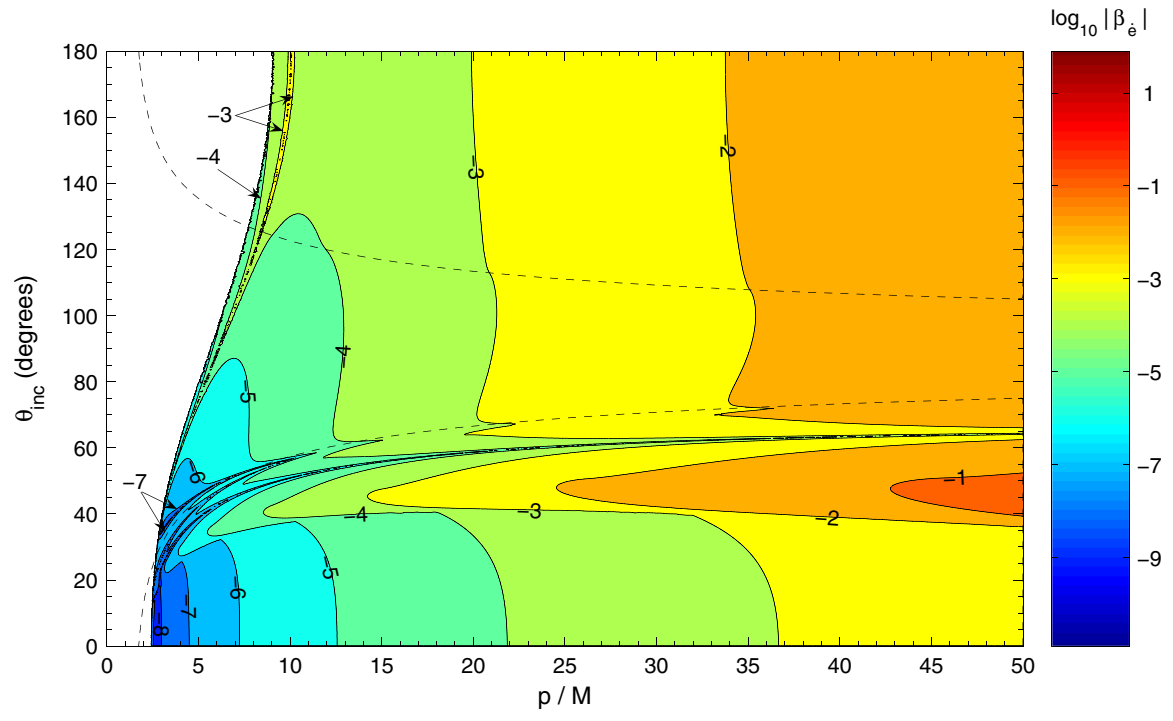

FIG. 5 (color online). The same as in Fig. 4, but for $\log _{10}\left|\beta_{\dot{e}}\right|$. 


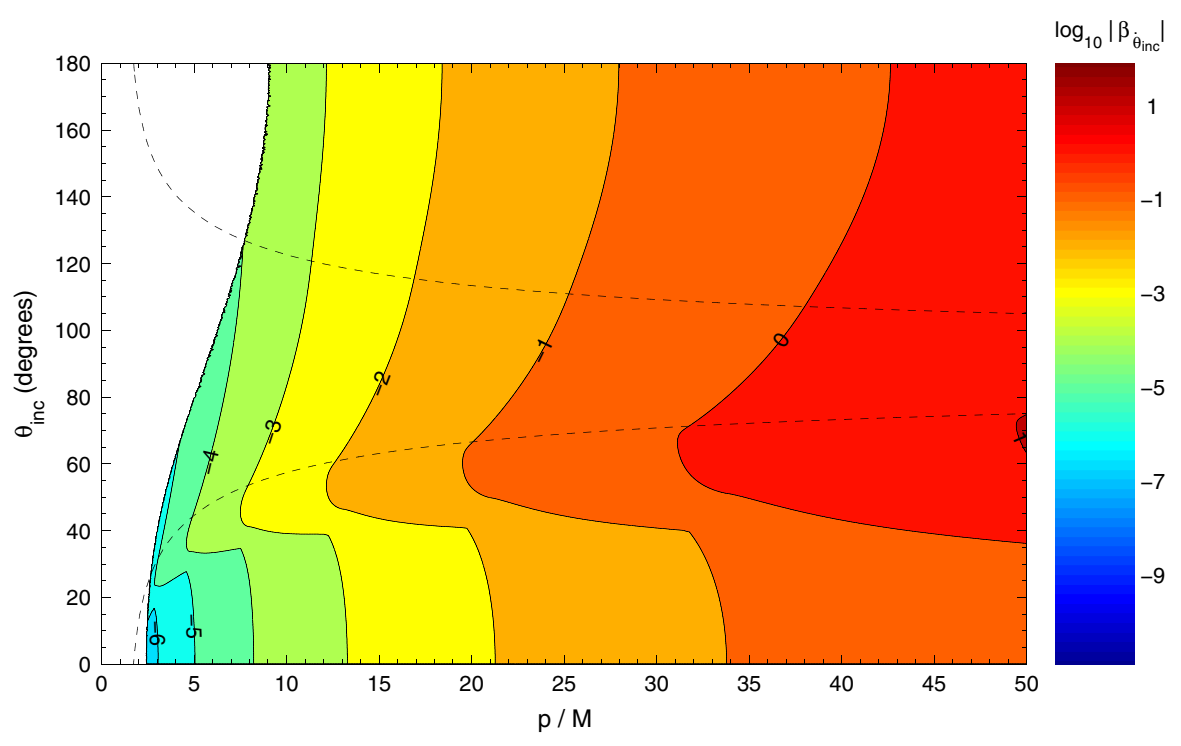

FIG. 6 (color online). The same as in Fig. 4, but for $\log _{10}\left|\beta_{\dot{\theta}_{\text {inc }}}\right|$.

Figure 4, for instance, shows $\left|\beta_{\dot{p}}\right|$ and indicates that the effect of the hydrodynamic drag is larger for orbits with high inclination, for any given semilatus rectum $p$. This is simply due to the fact that orbits with $\theta_{\text {inc }}>90$ degrees are retrograde with respect to the torus, and the velocity of the satellite relative to the fluid can easily become supersonic. Indeed this effect is visible also in the figures of Sec. IVA. (We recall that in those figures the retrograde orbits are mapped to negative values of the orbital radius $r$.) The transition between the subsonic and the supersonic regime is marked by the sharp bend of the contour levels of Fig. 4 at $\theta_{\text {inc }} \sim 40$ degrees. This bend corresponds to the passage from orbits which are always subsonic (the orbits with $\theta_{\text {inc }}$ smaller than the inclination angle at which the bend is located) to orbits which are supersonic at least for a part of their trajectory (the orbits with $\theta_{\text {inc }}$ larger than the inclination angle at which the bend is located). Another small dip is hardly noticeable in the contour levels at inclination angles $\theta_{\text {inc }}$ just smaller than the edge of the torus (and smaller than 90 degrees, corresponding therefore to prograde orbits); this feature corresponds to the transition from orbits which are partly subsonic and partly supersonic ("below" the dip), to orbits which keep always supersonic ("above" the dip). ${ }^{11}$ From Fig. 4 one can also

\footnotetext{
${ }^{11}$ We note that in order to better understand the fine features in the contour levels, we have built an auxiliary code computing the quantities $(d p / d t)_{\text {hydro }},(d e / d t)_{\text {hydro }}$, and $\left(d \cos \theta_{\text {inc }} / d t\right)_{\text {hydro }}$ by direct integration of Eqs. (43), (44), (47), and (50) along numerically solved geodesics, averaging over a reasonably large number of revolutions $(\sim 30)$ for each geodesic. This has not only validated the results which have been used to build the figures and which have been obtained using Eqs. (68), but has also allowed us to examine in detail the behavior of the geodesics in the various regions of the parameter space $\left(p, e, \theta_{\text {inc }}\right)$, thus helping to interpret the complicated features of the figures shown in this paper.
}

note that $\left|\beta_{\dot{p}}\right|$ becomes lower than $10^{-8}$ in a narrow "strip" at $p / M<5$. Indeed, $(d p / d t)_{\text {hydro }}$ changes sign inside this strip, being positive inside the region between the strip and the separatrix and negative outside, while $(d p / d t)_{\mathrm{GW}}$ is always negative. This behavior generalizes that of circular equatorial orbits, for which $(d r / d t)_{\text {hydro }}$ changes sign at the center of the torus (cf. Sec. IVA). Also in this case, however, the very small values of $\left|\beta_{\dot{p}}\right|$ cannot produce an observable imprint on the waveforms.

In a similar way, Fig. 5 shows the behavior of $\left|\beta_{\dot{e}}\right|$. As it can be seen, the influence of the torus is again larger at high inclinations than at low ones, for any fixed semilatus rectum. Also in this case, this happens because the orbits counterrotating with respect to the torus can easily become supersonic. As in Fig. 4, we can note the presence of a sharp bend in the contour levels at $\theta_{\text {inc }} \sim 40$ degrees, due to the transition from orbits always subsonic to orbits partly supersonic, and a dip in the contour levels near the edge of the torus (at inclinations $\theta_{\text {inc }}<90$ degrees), more pronounced than in Fig. 4 but again due to the transition from orbits only partly subsonic to orbits always supersonic. Moreover, one can note the presence of three "valleys" where the efficiency $\left|\beta_{\dot{e}}\right|$ becomes very small. One ("valley 1 ") starts at $\theta_{\text {inc }} \approx 15$ degrees, very close to the separatrix, and extends as far as the right edge of the figure $\left(p / M=50, \theta_{\text {inc }} \approx 65\right.$ degrees $)$ and beyond. A second valley ("valley 2 ") starts at the same point as valley 1 , but extends only until $p / M \approx 12$ and $\theta_{\text {inc }} \approx 60$ degrees, where it terminates together with a third valley ("valley 3 ") starting on the separatrix at $\theta_{\text {inc }} \approx 30$ degrees. Across these valleys, the quantity $(d e / d t)_{\text {hydro }}$ becomes zero and changes sign, being negative under valley 1 and in the region between the separatrix and valleys 2 and 3, and positive in the rest of the $\left(p, \theta_{\text {inc }}\right)$ plane. Conversely, the rate of change of the eccentricity due to radiation reaction 


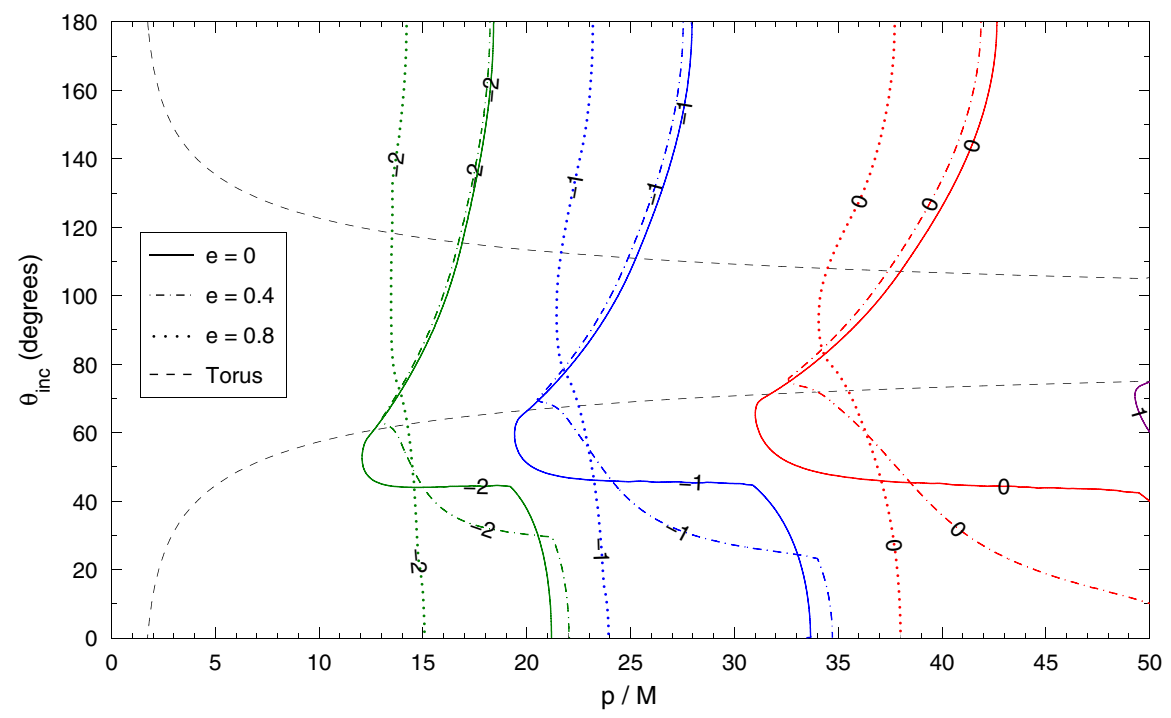

FIG. 7 (color online). $\quad \log _{10}\left|\beta_{\dot{\theta}_{\text {inc }}}\right|$ shown as a function of the semilatus rectum $p$ and of the inclination angle $\theta_{\text {inc }}$ for inclined orbits with selected values of the eccentricity, i.e., $e=0$ (solid line), $e=0.4$ (dot-dashed line), $e=0.8$ (dotted line). The figure refers to the model A1 of Table I, but a larger (smaller) mass $M_{\mathrm{t}}$ for the torus would simply increase (decrease) $\left|\beta_{\dot{\theta}_{\text {inc }}}\right|$ by a factor $M_{\mathrm{t}} /(0.1 M)$. The dashed line marks the edge of the torus.

is always negative, with the exception of orbits very close to the separatrix [52]; this is apparent also in Fig. 5, where the narrow strip corresponding to a ratio $\left|\beta_{\dot{e}}\right| \gtrsim 10^{-3}$ and running close and almost parallel to the separatrix is due to a change in sign of $(d e / d t)_{\mathrm{GW}}$. Despite this markedly different behavior of $(d e / d t)_{\text {hydro }}$ and $(d e / d t)_{\mathrm{GW}}$, Fig. 5 shows that the effect of the hydrodynamic drag is always much smaller than radiation reaction unless the semilatus rectum of the orbit is increased to $p / M \gtrsim 50$, or the mass of the torus is increased at least by a factor 10 thus extrapolating to $M_{\mathrm{t}}=M .{ }^{12}$ However, while a larger semilatus rectum increases the efficiency $\beta_{\dot{e}}$, it also reduces the frequency and amplitude of the gravitational-wave signal, moving it to a region of low sensitivity for LISA.

The effect of the hydrodynamic drag is somewhat stronger when considered in terms of the efficiency $\left|\beta_{\dot{\theta}_{\text {inc }}}\right|$, as it is shown in Fig. 6. While the qualitative behavior is similar to the one discussed for the two preceding figures, it should be noted that the effect of the torus is comparable to that of radiation reaction already for $p / M \approx 32$ if $\theta_{\text {inc }} \approx 60$ degrees, and the two effects remain comparable down to $p / M \approx 20$ if the mass of the torus is increased by a factor 10 thus extrapolating to $M_{\mathrm{t}}=M$. Moreover, while radiation reaction produces an increase in the inclination $\theta_{\text {inc }}$ irrespective of the orbital parameters [36], $\left(d \theta_{\text {inc }} / d t\right)_{\text {hydro }}$ is always negative and thus a measurement of the evolution of the inclination angle $\theta_{\text {inc }}$ in the early stages of an EMRI could give important information on the presence of a torus. If such a presence were to be

\footnotetext{
${ }^{12}$ The test-fluid approximation of course breaks down in this limit.
}

detected, it would not prevent high-precision tests of the Kerr nature of the SMBH being performed in the strongfield region, where the hydrodynamic drag becomes negligible.

We should also note that the decrease of $\theta_{\text {inc }}$ due to the hydrodynamic drag is not surprising for orbits with $\theta_{\text {inc }}>$ 90 degrees (i.e., orbits rotating in the opposite $\phi$ direction with respect to the fluid), because the hydrodynamic drag clearly induces the orbits to rotate in the same $\phi$ direction as the torus. For orbits with $\theta_{\text {inc }}<90$ degrees, instead, the decrease of $\theta_{\text {inc }}$ comes directly from Eq. (45) (we recall that the effect of the radial drag averages out when adopting the adiabatic approximation), thus following directly from the axis and plane symmetry of the system and being independent of the use of constant specific angular momentum tori such as the ones considered in this paper. Indeed, since the fluid of the torus does not move in the $\theta$ direction, Eq. (45) states that accretion conserves the momentum of the satellite in the $\theta$ direction, but it also increases its mass, thus reducing the velocity in the $\theta$ direction. In addition, the dynamical friction will contribute to this reduction by damping further the oscillations around the equatorial plane.

To illustrate how the above results depend on the eccentricity, we show in Fig. 7 the efficiency $\left|\beta_{\dot{\theta}_{\text {inc }}}\right|$ for the model A1 of Table I, but for different values of the eccentricity i.e., $e=0,0.4$, and 0.8. (Equivalent figures could be made also for $\left|\beta_{\dot{p}}\right|$ and $\left|\beta_{\dot{e}}\right|$, but we omit them here because they would be qualitatively similar to Fig. 7.) For each value of the eccentricity, we have computed $\left|\beta_{\dot{\theta}_{\text {inc }}}\right|$ for $\sim 4 \times 10^{4}$ orbits, and using the same technique employed for Figs. 46 we have drawn the contours corresponding to values of 
$0.01,0.1,1$, and 10. Also in this case, a larger (smaller) mass $M_{\mathrm{t}}$ for the torus would simply increase (decrease) these absolute ratios by a factor $M_{\mathrm{t}} /(0.1 M)$.

Clearly, many of the features in this plot have been discussed also for the previous figures. For instance, the contour levels present sharp bends at low inclinations (i.e., $\theta_{\mathrm{inc}} \approx 10-40$ degrees) for $e=0$ and $e=0.4$, due the transition from subsonic to partly supersonic orbits, whereas the transition from partly supersonic to fully supersonic orbits causes the appearance of a pronounced "kink" in the $e=0.4$ contour levels, for inclinations $\theta_{\text {inc }}<90$ degrees just above the edge of the torus. The $e=$ 0.8 contour levels, on the other hand, are rather smooth and less affected by the complex changes of regimes as the satellite interacts with the torus. Most importantly, however, Fig. 7 suggests that the conclusions drawn when discussing Fig. 6 for orbits with $e=0.1$ are not altered significantly by a change in the eccentricity. Indeed, even for large eccentricities the influence of the torus on the evolution of $\theta_{\text {inc }}$ can be comparable to that of radiation reaction for $p / M$ as small as 35-38, while the two effects are still equal at $p / M \approx 23-24$ if the mass of the torus is increased by a factor 10 thus extrapolating to $M_{\mathrm{t}}=M$. As a result, a measurement of the evolution of the inclination angle $\theta_{\text {inc }}$ even for generic eccentric orbits could give important information on the presence of a torus around the SMBH.

This conclusion is finally confirmed by Fig. 8, in which we show how $\left|\beta_{\dot{\theta}_{\text {inc }}}\right|$ changes if one considers different values of the outer radius of the torus while keeping its mass fixed. More specifically, Fig. 8 shows the location in the $\left(p, \theta_{\text {inc }}\right)$ plane of the circular orbits for which $\left|\beta_{\dot{\theta}_{\text {inc }}}\right|=$ 1. Different curves refer to different values of the outer radius, and, in particular, to the models $\mathrm{A} 1, \mathrm{C} 1, \mathrm{C} 3$ and $\mathrm{C} 5$ of Table I. As it is probably obvious by now, a different mass $M_{\mathrm{t}}$ for the torus would simply make the curves of this figure correspond to $\left|\beta_{\dot{\theta}_{\text {inc }}}\right|=M_{\mathrm{t}} /(0.1 M)$. As expected from the results of Sec. IVA, a modest variation of the outer radius can easily cause the decrease of the inclination angle due to the hydrodynamic drag to be dominant over the increase due to radiation reaction for orbits with $p / M \sim 20$ or smaller.

\section{CONCLUSIONS}

SMBHs are expected to be surrounded by matter, either in the form of stellar disks, as in the case of normal galactic centers, or in the form of accretion disks of gas and dust, as in the case of AGNs. In order to assess under what conditions and to what extent the interaction with matter could modify the gravitational-wave signal from EMRIs in AGNs, we have studied EMRIs in spacetimes containing a SMBH surrounded by a non-self-gravitating torus. For simplicity, and in order to handle the equilibrium solution analytically, we have considered a torus with a constant distribution of specific angular momentum, using as reference dimensions and masses those for the accretion disks expected in AGNs, but bearing in mind that these also come with rather large uncertainties. We have extrapolated our results also to cases in which the mass of the torus is comparable with that of the SMBH, although we stress that in this limit our test-fluid approximation for the torus is no longer valid.

Overall, we have found that the effect of the hydrodynamic drag exerted by the torus on the satellite black hole can have important effects sufficiently far from the central object, and that these effects are qualitatively differ-

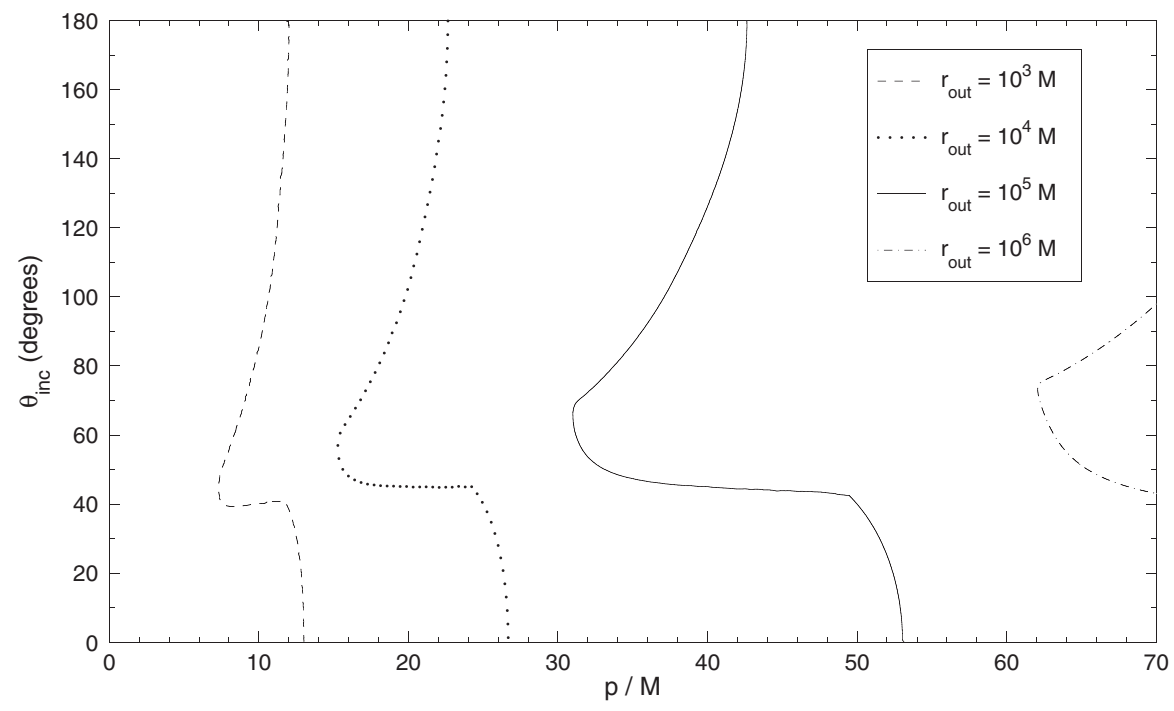

FIG. 8. Location in the $\left(p, \theta_{\text {inc }}\right)$ plane of the circular orbits for which $\left|\beta_{\dot{\theta}_{\text {inc }}}\right|=1$. Different curves refer to different values of the outer radius and thus to models $\mathrm{A} 1, \mathrm{C} 1, \mathrm{C} 3$ and $\mathrm{C} 5$ of Table $\mathrm{I}$. Note that a different mass $M_{\mathrm{t}}$ for the torus would simply make the curves of this figure to correspond to $\left|\beta_{\dot{\theta}_{\text {inc }}}\right|=M_{\mathrm{t}} /(0.1 M)$. 
ent from those of radiation reaction. In particular, if the torus is corotating with the $\mathrm{SMBH}$, the hydrodynamic drag always decreases the inclination of the orbits with respect to the equatorial plane (i.e., orbits evolve towards the equatorial prograde configuration), whereas radiation reaction always increases the inclination (i.e., orbits evolve towards the equatorial retrograde configuration). In the case of a system composed of a SMBH with mass $M=$ $10^{6} M_{\odot}$ and a corotating torus with mass $M_{\mathrm{t}} \lesssim M$, the effect of the torus will be marginally observable by LISA only if the radius of the torus is as small as $r_{\text {out }} \approx$ $10^{3}-10^{4} M$. However, if the SMBH has a lower mass, EMRIs will be detectable by LISA at larger distances from the SMBH, and the effects of a torus will be more evident. For instance, for a SMBH with $M=10^{5} M_{\odot}$ and a corotating torus with outer radius $r_{\text {out }}=10^{5} \mathrm{M}$ and mass $M_{\mathrm{t}}=0.1 M\left(M_{\mathrm{t}}=M\right)$, the inclination with respect to the equatorial plane will decrease, due to the hydrodynamic drag, for orbits with semilatus rectum $p \gtrsim 35 M$ ( $p \gtrsim$ $25 M$ ), while the EMRI signal will start being detectable by LISA already at a distance of $\approx 50 M$ from the SMBH. Note, however, that unless one considers as the satellite an intermediate-mass black hole with $m \sim 100 M_{\odot}$ around a $10^{5} M_{\odot} \mathrm{SMBH}$ (a configuration which may be possible but about which too little is yet known), considering EMRIs at such large distances from the SMBH has the obvious drawback that the amplitude of the gravitational-wave signal will be proportionally smaller. This will considerably reduce the detection volume, although the decrease in the event rate could be mitigated by the fact that weak-field EMRIs are probably more numerous than strong-field EMRIs, which are the ones accounted for in standard calculations of event rates.

In general, we expect measurements of the evolution of the inclination angle in the early phases of EMRIs to be a potential source of important information about the presence of thick tori which could not be detected by other techniques. Moreover, because for any astrophysically plausible torus configurations the effect of the hydrodynamic drag becomes rapidly negligible in the very strongfield region of the SMBH (i.e., $p \lesssim 5 M$ ), the presence of a torus would not prevent high-precision tests of the Kerr nature of the SMBH being performed.

Although obtained with a simple model for the torus (i.e., with a constant specific angular momentum), the important feature that distinguishes the hydrodynamic drag from radiation reaction, namely, the decrease of the inclination angle, cannot be affected by a change of the specific angular momentum distribution (we recall that $\ell$ must be increasing with radius for stability). Such a feature, in fact, is simply due to the conservation of the momentum of the satellite in the $\theta$ direction during accretion and to the dynamical friction of the fluid: both effects force the satellite to smaller inclinations by reducing its $\theta$ velocity. However, the calculation of the magnitude of the hydrodynamic drag and how it compares with radiation reaction for more general disk models is not straightforward.

Tori built with increasing distributions of specific angular momentum, in fact, would have two substantial differences with respect to those considered here. First, the separation between the specific angular momentum of the torus and the Keplerian specific angular momentum will generally decrease for orbits corotating with the torus, thus reducing the relative motion between the satellite and the fluid and consequently the hydrodynamic drag, whereas it will increase for orbits counterrotating relative to the torus, thus enhancing the hydrodynamic drag. The magnitude of this effect depends on the precise angular momentum distribution considered and rough estimates can be made assuming a power law for the specific angular momentum, i.e., $\ell / M \sim(r / M)^{\alpha}$, with $\alpha<0.5$ for the torus to have an outer radius and a cusp [37,39]. Using the general formulas reported in Sec. II, it is easy to check that for $r$ between $20 M$ and $50 M$ and prograde orbits, the relative motion decreases by $\sim 20-30 \%$ for $\alpha=0.1$ and by at least $95 \%$ for $\alpha=0.4$ (this significant decrease is due to the fact that for $\alpha=0.4$ the center moves to a radius $r_{\text {center }} \sim 27 M$, just in the middle of the radial interval which we are considering). Conversely, in the same radial range the increase for counterrotating orbits is of about $8-10 \%(30-45 \%)$, for $\alpha=0.1(0.4)$. Second, the density in the inner parts of the torus will generally decrease. Using again the expressions in Sec. II, it is easy to check that the density decreases by about $18 \%$ (97\%) at $r \sim 20 M$ and by about $11 \%(90 \%)$ at $r \sim 50 M$, for $\alpha=0.1(0.4)$.

Overall, therefore, the decrease of the inclination angle due to the hydrodynamic drag could be detectable by LISA also for nonconstant $\ell$ tori, especially if $\ell$ varies slowly with the radius or, if $\ell$ varies rapidly with the radius, if the EMRI is counterrotating relative to the torus.

Finally, let us comment on two further effects that can in principle occur in the systems considered in this paper. First, the motion of the satellite will be influenced by the gravitational attraction exerted by the torus. This is clearly a conservative effect, and cannot therefore influence the infall of the satellite towards the SMBH, which is instead regulated by the dissipative forces (radiation reaction and hydrodynamic drag). However, this effect can in principle cause the periastron to advance, thus introducing a phase shift in the gravitational waveforms. (Note that a similar advance is caused by the conservative part of the gravitational self-force [62].) To calculate the order of magnitude of this effect, let us consider for simplicity a thin disk with outer radius $r_{\text {out }}$, mass $M_{\mathrm{D}}$, and constant surface density, and a satellite of mass $m$ located on the equatorial plane at a distance $d \ll r_{\text {out }}$ from the central SMBH, the mass of which we denote by $M$. The potential energy of the satellite due to the gravitational field of the disk can be easily calculated to be, up to a constant and to leading order, 


$$
U \approx \frac{m M_{\mathrm{D}} d^{2}}{2 r_{\mathrm{out}}^{3}} .
$$

This potential energy can be used to compute the Newtonian periastron precession of the satellite's orbit during a revolution [use for instance Eq. (1) of Ref. [63], chapter 3, exercise number 3]:

$$
\delta \phi \approx-\frac{3 M_{\mathrm{D}} \pi d^{3}}{M r_{\mathrm{out}}^{3}}
$$

for almost circular orbits. Using this equation and the wellknown Newtonian formula for the revolution period, it is easy to check that, for orbits relevant for LISA, the total phase shift accumulated in $1 \mathrm{yr}$ is $\ll 2 \pi$ as long as $r_{\text {out }} \gtrsim$ $10^{4} M$ and $M=10^{5}-10^{6} M_{\odot}$. Because LISA is not expected to detect phase lags less than 1 cycle over its lifetime, ${ }^{13}$ this periastron advance and the consequent phase

\footnotetext{
${ }^{13}$ This corresponds indeed to a dephasing time of the order of LISA's lifetime.
}

shift cannot be observed. On the other hand, for disks or tori with $r_{\text {out }} \sim 10^{3} \mathrm{M}$, this effect could in principle be marginally visible by LISA (especially if $M=10^{5} M_{\odot}$ ).

A second effect which could in principle affect EMRIs in the presence of a torus is the spin of the satellite black hole, which increases due to accretion of the torus material. The satellite's spin couples with the orbital angular momentum as well as with the spin of the SMBH, but its effect on the motion is negligible over a time scale of $1 \mathrm{yr}$ [64], unless it is close to its maximal value (in which case it might be marginally observable) [64].

\section{ACKNOWLEDGMENTS}

It is a pleasure to thank L. Barack and J.C. Miller for giving helpful advice and comments on this manuscript, $\mathrm{P}$. Montero for insightful comments about the torus solutions that we use in this paper, as well as L. Subr and V. Karas for very useful remarks about the hydrodynamic interaction between a moving body and a fluid.
[1] http://lisa.nasa.gov/; http://sci.esa.int/home/lisa/.

[2] J. Kormendy and D. Richstone, Annu. Rev. Astron. Astrophys. 33, 581 (1995).

[3] J. R. Gair, L. Barack, T. Creighton, C. Cutler, S. L. Larson, E.S. Phinney, and M. Vallisneri, Classical Quantum Gravity 21, S1595 (2004).

[4] E. Poisson, Living Rev. Relativity 7, 6 (2004); E. Poisson, arXiv:gr-qc/0410127.

[5] M. Colpi, S. L. Shapiro, and I. Wasserman, Phys. Rev. Lett. 57, 2485 (1986); D. F. Torres, S. Capozziello, and G. Lambiase, Phys. Rev. D 62, 104012 (2000).

[6] F. Munyaneza, D. Tsiklauri, and R. D. Viollier Astrophys. J. Lett. 509, L105 (1998); R. Schodel et al., Nature (London) 419, 694 (2002).

[7] P. O. Mazur and E. Mottola, Proc. Natl. Acad. Sci. U.S.A. 101, 9545 (2004); A.E. Broderick and R. Narayan, Astrophys. J. 638, L21 (2006); C. B. M. Chirenti and L. Rezzolla, Classical Quantum Gravity 24, 4191 (2007).

[8] F. D. Ryan, Phys. Rev. D 52, 5707 (1995); 56, 1845 (1997); 56, 7732 (1997).

[9] M. Kesden, J. Gair, and M. Kamionkowski, Phys. Rev. D 71, 044015 (2005)

[10] N. A. Collins and S. A. Hughes, Phys. Rev. D 69, 124022 (2004).

[11] K. Glampedakis and S. Babak, Classical Quantum Gravity 23, 4167 (2006); L. Barack and C. Cutler, Phys. Rev. D 75, 042003 (2007).

[12] Y. Levin and A. M. Beloborodov, Astrophys. J. Lett. 590, L33 (2003); R. Genzel et al., Astrophys. J. 594, 812 (2003).

[13] R. Antonucci, Annu. Rev. Astron. Astrophys. 31, 473 (1993); C. M. Urry and P. Padovani, Publ. Astron. Soc.
Pac. 107, 803 (1995).

[14] S. Collin and J.M. Huré, Astron. Astrophys. 372, 50 (2001); J. M. Huré, Astron. Astrophys. 395, L21 (2002).

[15] E. Barausse, L. Rezzolla, D. Petroff, and M. Ansorg, Phys. Rev. D 75, 064026 (2007).

[16] H. Bondi and F. Hoyle, Mon. Not. R. Astron. Soc. 104, 273 (1944); H. Bondi, Mon. Not. R. Astron. Soc. 112, 195 (1952).

[17] L. I. Petrich, S.L. Shapiro, R. F. Stark, and S. A. Teukolsky, Astrophys. J. 336, 313 (1989).

[18] J. A. Font and J. M. Ibanez, Astrophys. J. 494, 297 (1998).

[19] J. A. Font and J. M. Ibanez, Mon. Not. R. Astron. Soc. 298, 835 (1998); J. A. Font, J. M. Ibanez, and P. Papadopoulos, Mon. Not. R. Astron. Soc. 305, 920 (1999).

[20] S. Chandrasekhar, Astrophys. J. 97, 255 (1943).

[21] Y. Rephaeli and E.E. Salpeter, Astrophys. J. 240, 20 (1980); M. A. Ruderman and E. A. Spiegel, Astrophys. J. 165, 1 (1971).

[22] E. C. Ostriker, Astrophys. J. 513, 252 (1999).

[23] F. J. Sanchez-Salcedo and A. Brandenburg, Astrophys. J. 522, L35 (1999).

[24] H. Kim and W. T. Kim, Astrophys. J. 665, 432 (2007).

[25] E. Barausse, Mon. Not. R. Astron. Soc. 382, 826 (2007).

[26] D. Vokrouhlicky and V. Karas, Mon. Not. R. Astron. Soc. 265, 365 (1993).

[27] D. Vokrouhlicky and V. Karas, Mon. Not. R. Astron. Soc. 298, 53 (1998); L. Subr and V. Karas, Astron. Astrophys. 352, 452 (1999).

[28] V. Karas and L. Subr, Astron. Astrophys. 376, 686 (2001).

[29] R. Narayan Astrophys. J. 536, 663 (2000).

[30] R.D. Blandford and M.C. Begelman, Mon. Not. R. Astron. Soc. 303, L1 (1999). 
[31] Y. Levin, Mon. Not. R. Astron. Soc. 374, 515 (2007); Y. Levin, arXiv:astro-ph/0307084.

[32] S. K. Chakrabarti, Astrophys. J. 411, 610 (1993); Phys. Rev. D 53, 2901 (1996).

[33] M. Kozlowski, M. Jaroszynski, and M. A. Abramowicz, Astron. Astrophys. 63, 209 (1978).

[34] M. A. Abramowicz, M. Jaroszynski, and M. Sikora, Astron. Astrophys. 63, 221 (1978).

[35] F. H. Seguin, Astrophys. J. 197, 745 (1975).

[36] F. D. Ryan, Phys. Rev. D 52, R3159 (1995); S. A. Hughes, Phys. Rev. D 61, 084004 (2000); E. Barausse, S. A. Hughes, and L. Rezzolla, Phys. Rev. D 76, 044007 (2007).

[37] J. A. Font and F. Daigne, Mon. Not. R. Astron. Soc. 334, 383 (2002); F. Daigne and J. A. Font, Mon. Not. R. Astron. Soc. 349, 841 (2004).

[38] O. Zanotti, L. Rezzolla, and J. A. Font, Mon. Not. R. Astron. Soc. 341, 832 (2003).

[39] O. Zanotti, J. A. Font, L. Rezzolla, and P. Montero, Mon. Not. R. Astron. Soc. 356, 1371 (2005).

[40] C. W. Misner, K. S. Thorne, and J. A. Wheeler, Gravitation (Freeman, San Francisco, 1973).

[41] S. L. Shapiro and S. A. Teukolsky, Black Holes, White Dwarfs, and Neutron Stars: The Physics of Compact Objects (Wiley, New York, 1983).

[42] S. Drasco, E. E. Flanagan, and S. A. Hughes, Classical Quantum Gravity 22, S801 (2005).

[43] Y. Mino, Phys. Rev. D 67, 084027 (2003).

[44] S. A. Hughes, S. Drasco, E. E. Flanagan, and J. Franklin, Phys. Rev. Lett. 94, 221101 (2005).

[45] S. Drasco and S. A. Hughes, Phys. Rev. D 73, 024027 (2006).

[46] B. Carter, Phys. Rev. 174, 1559 (1968).

[47] D. Kennefick and A. Ori, Phys. Rev. D 53, 4319 (1996).

[48] F. D. Ryan, Phys. Rev. D 53, 3064 (1996).

[49] W. Schmidt, Classical Quantum Gravity 19, 2743 (2002).
[50] S. Drasco and S. A. Hughes, Phys. Rev. D 69, 044015 (2004).

[51] W. H. Press, S. A. Teukolsky, W. T. Vetterling, and B. P. Flannery, Numerical Recipes (Cambridge University Press, Cambridge, England, 1992).

[52] J. R. Gair and K. Glampedakis, Phys. Rev. D 73, 064037 (2006).

[53] S. A. Teukolsky, Astrophys. J. 185, 635 (1973); M. Sasaki and T. Nakamura, Prog. Theor. Phys. 67, 1788 (1982).

[54] K. S. Thorne, Astrophys. J. 191, 507 (1974).

[55] L. Rezzolla, P. Diener, E. N. Dorband, D. Pollney, C. Reisswig, E. Schnetter, and J. Seiler, arXiv:gr-qc/ 0708.3999.

[56] L. Rezzolla, E. N. Dorband, C. Reisswig, P. Diener, D. Pollney, E. Schnetter, and B. Szilagyi, Astrophys. J. 674, L29 (2008).

[57] P. L. Bender and D. Hils, Classical Quantum Gravity 14, 1439 (1997); G. Nelemans, L. R. Yungelson, and S.F. Portegies Zwart, Astron. Astrophys. 375, 890 (2001); A. J. Farmer and E.S. Phinney, Mon. Not. R. Astron. Soc. 346, 1197 (2003).

[58] P. C. Peters and J. Mathews, Phys. Rev. 131, 435 (1963); L. Barack and C. Cutler, Phys. Rev. D 69, 082005 (2004).

[59] P. Amaro-Seoane, J. R. Gair, M. Freitag, M. Coleman Miller, I. Mandel, C. J. Cutler, and S. Babak, Classical Quantum Gravity 24, R113 (2007).

[60] S. Drasco, Classical Quantum Gravity 23, S769 (2006).

[61] K. Glampedakis and D. Kennefick, Phys. Rev. D 66, 044002 (2002).

[62] A. Pound, E. Poisson, and B. G. Nickel, Phys. Rev. D 72, 124001 (2005).

[63] L. D. Landau and E. M. Lifshitz, Mechanics (Pergamon, New York, 1976).

[64] L. Barack and C. Cutler, Phys. Rev. D 69, 082005 (2004). 